\title{
Advanced Strained-Superlattice Photocathodes for Polarized Electron Source
}

\author{
Final Report \\ Contract \# DE-FG02-01ER83332
}

Submitted by:

Aaron Moy

SVT Associates

Eden Prairie, MN 


\section{Table of Contents}

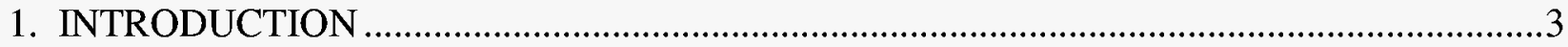

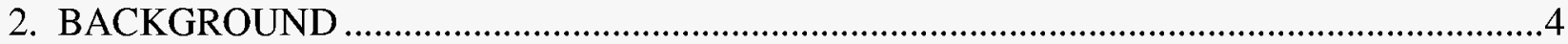

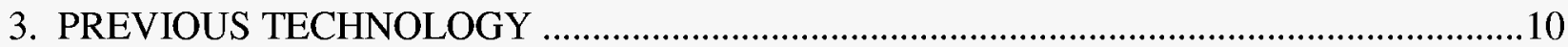

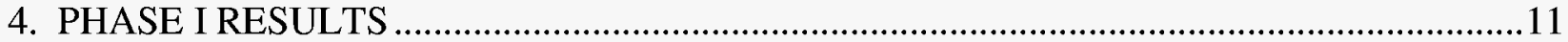

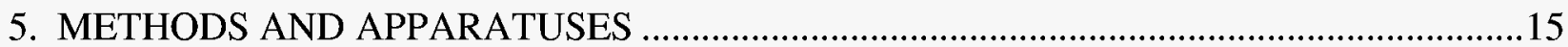

6. INITIAL COMPARISON WITH PREVIOUS PHOTOCATHODES ...............................18

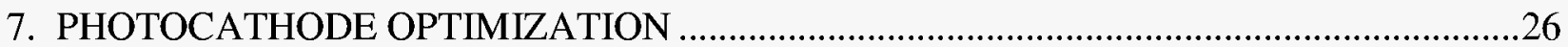

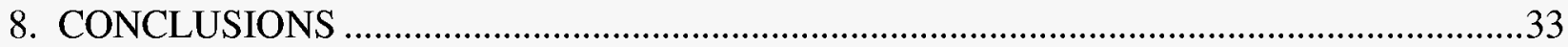

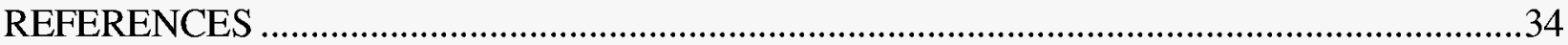




\section{INTRODUCTION}

Polarized electrons have been essential for high-energy parity-violating experiments and measurements of the nucleon spin structure. The availability of a polarized electron beam was crucial to the success of the Stanford Linear Collider (SLC) in achieving a precise measurement of the electroweak mixing angle, and polarized electron beams will be required for all future linear colliders. Polarized electrons are readily produced by GaAs photocathode sources. When a circularly polarized laser beam tuned to the bandgap minimum is directed to the negativeelectron-affinity (NEA) surface of a GaAs crystal, longitudinally polarized electrons are emitted into vacuum. The electron polarization is easily reversed by reversing the laser polarization. The important properties of these photocathodes for accelerator applications are: degree of polarization of the extracted beam; ability to extract sufficient charge to meet accelerator pulsestructure requirements; efficiency and stability of operation; and absence of any asymmetries in the beam properties (charge, position, energy, etc.) upon polarization reversal.

The performance of GaAs photocathodes has improved significantly since they were first introduced in 1978 [1]. The theoretical maximum polarization of 50\% for natural GaAs was first exceeded in 1991 using the lattice mismatch of a thin InGaAs layer epitaxially grown over a GaAs substrate to generate a strain in the former that broke the natural degeneracy between the heavy- and light-hole valence bands [2]. Polarizations as high as 78\% were produced for the SLC from photocathodes based on a thin GaAs epilayer grown on GaAsP $[3,4]$.

After 10 years of experience with many cathode samples at several laboratories [5], the maximum polarization using the GaAs/GaAsP single strained-layer cathode remained limited to $80 \%$, while the quantum efficiency $(\mathrm{QE})$ for a $100-\mathrm{nm}$ epilayer is only $0.3 \%$ or less. Two factors were known to limit the polarization of these cathodes: 1) the limited band splitting; and 2) a relaxation of the strain in the epilayer since the 10-nm critical thickness for maintaining perfect strain is exceeded for a $1 \%$ lattice-mismatch [6]. Strained superlattice structures, consisting of very thin quantum well layers alternating with lattice-mismatched barrier layers are excellent candidates for higher polarization. Due to the difference in the effective mass of the heavy- and light-holes, a superlattice exhibits a natural splitting of the valence band, which adds to the strain-induced splitting. In addition, each of the SL layers is thinner than the critical thickness. Polarized photoemission from strained InGaAs/GaAs [7], InGaAs/AlGaAs [8], and GaAs/GaAsP [9,10] superlattice structures have been reported in the literature.

For this Phase II program, SVT Associates worked with the Stanford Linear Accelerator Center (SLAC) and University of Wisconsin at Madison to create photocathodes with improved polarization by employing GaAs/GaAsP superlattices. These superlattices consist of alternating thin layers of GaAs and GaAsP. The thicknesses and alloy compositions are designed to create a strained GaAs photoemission layer. Under strain, the heavy-hole and light-hole valence bands in GaAs split, removing degeneracy and allowing high polarization, theoretically $100 \%$. This final report discusses the efforts and results achieved, comparing the device performance of newly created superlattice photocathodes grown by molecular beam epitaxy (MBE) with the devices created by other fabrication technologies, and efforts to optimize and improve the device operation. 


\section{BACKGROUND}

Polarized electron sources have wide applications in many branches of physics. Their use in linear accelerators generally requires high-intensity sources. For example, the Stanford Linear Collider (SLC) requires peak currents of about 8 amps in a $2.5 \mathrm{nsec}$ pulse at $120 \mathrm{~Hz}$. These requirements can be met using photoemission from negative-electron-affinity (NEA) GaAs, and this is the technique adopted for linear electron accelerators. Polarized electron sources based on NEA-GaAs were pioneered at SLAC. The original PEGGY II source produced $2 \times 10^{11} \mathrm{e}$ - per bunch at $120 \mathrm{~Hz}$ with an average spin polarization of $37 \%$ [1]. The maximum polarization of this type of source was limited to $50 \%$ due to the valence-band degeneracy of the heavy-hole and light-hole bands of GaAs.

A breakthrough development was achieved in 1991 by collaborators from SLAC, the University of Wisconsin, and the University of California-Berkeley who observed $71 \%$ spin-polarization from an InGaAs layer epitaxially grown on a GaAs buffer [2]. The observed polarization enhancement is a result of strain in the InGaAs layer due to a small $(0.9 \%)$ lattice mismatch of the epitaxial layer with the GaAs substrate. Strain induces valence-band splitting, which permits optical excitation of a single-band transition, thus potentially leading to $100 \%$ polarization of the photoemitted electrons. Although this pioneering experiment demonstrated strain-enhanced electron-spin polarization, the InGaAs-GaAs structure was not practical for a high brightness electron source due to its low quantum efficiency $(\mathrm{QE})$ and short cathode lifetime. To overcome this deficiency of the InGaAs-GaAs structure, the research shifted to strained GaAs epitaxially grown on GaAsP [4]. Electron-spin polarization as high as $85 \%$ was observed, and quantum efficiency reached $0.2 \%$. Strained GaAs photocathodes have been used exclusively in the SLAC polarized electron source since 1993, producing highly polarized electron beam for the SLC and fixed target high energy experiments [5]. Figure 2.1 shows the strained GaAs structure.

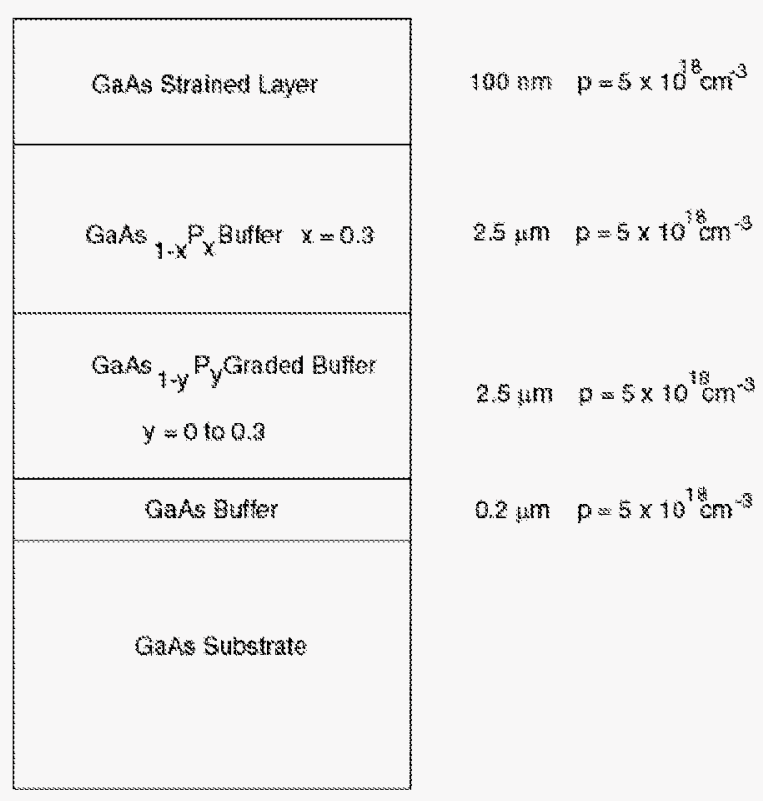

\section{Figure 2.1}

Polarized photocathode structure using strained GaAs. 
Despite these successes, the proposed Next Linear Collider (NLC) requires a much higher beam intensity. The present generation of strained GaAs is incapable by a factor of 20 of producing the required intensity.

To achieve higher polarization and higher charge output, we developed strained-superlattice photocathodes based on GaAs and GaAsP. Figure 2.2 shows the strained-superlattice structure. In this structure, the single-strained GaAs layer in Figure 2.1 is replaced by the strained-superlattice structure. The GaAsP layers are potential energy "barriers." The GaAs layers are "wells" and are strained.

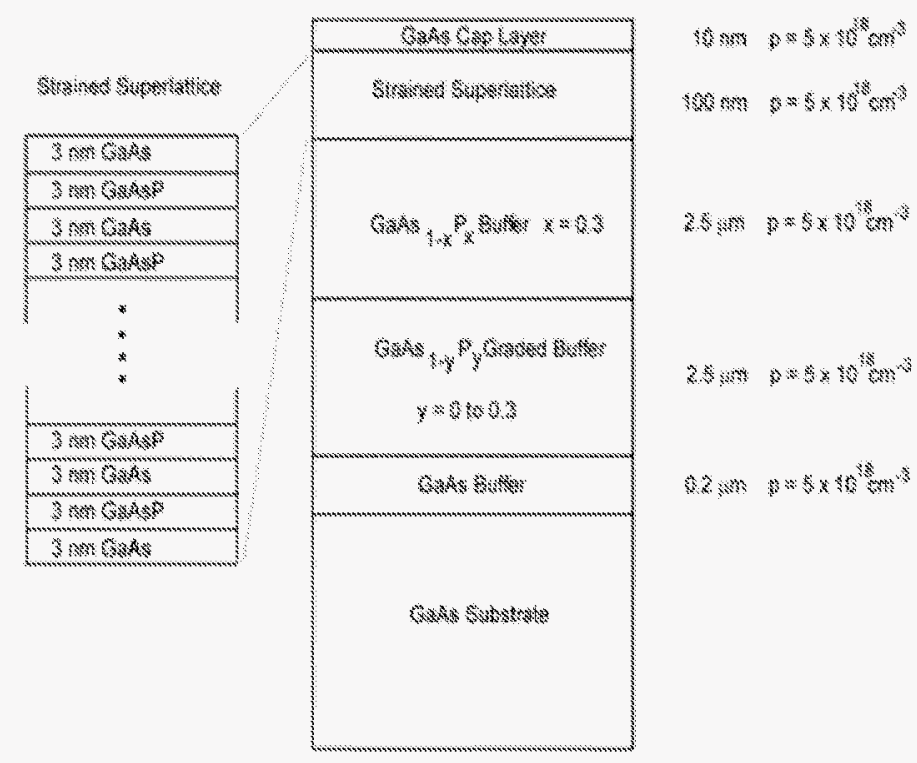

Figure 2.2.

Polarized photocathode structure using strained GaAs on top of a superlattice buffer layer.

The strained-superlattice structure is thought to increase the polarization because the valence band energy splitting is increased by the strain as well as the quantum-size-effect in superlattice. Charge enhancement is also attributed to the miniband in the superlattice structure that boosts the energy of the conduction band electrons, resulting in reduced surface electron trapping, which is responsible for the charge limit effect.

\section{Background Physics of the Approach}

The polarized electron source is based on photoemission of polarized electrons from negativeelectron-affinity III-V compounds such as GaAs. Electron spin-polarization is a consequence of the crystal symmetry of GaAs in that transitions from the top of the normally filled valence band to the bottom of the normally unoccupied conduction band are like transitions from the $j=3 / 2$ to the $\mathrm{j}=1 / 2$ state. Figure 2.3 shows the band structure and transitions for unstrained $\mathrm{GaAs}$ at $\mathrm{k}=0$. When unstrained GaAs is excited by circularly polarized light, two transitions between the valence band and conduction band $(\mathrm{C})$ are allowed due to the band degeneracy between the heavy-hole $(\mathrm{HH})$ and light-hole energy bands $(\mathrm{LH})$, with three times as many electron spins antiparallel to the photon spin as parallel to it, resulting in a polarization of $50 \%$. 


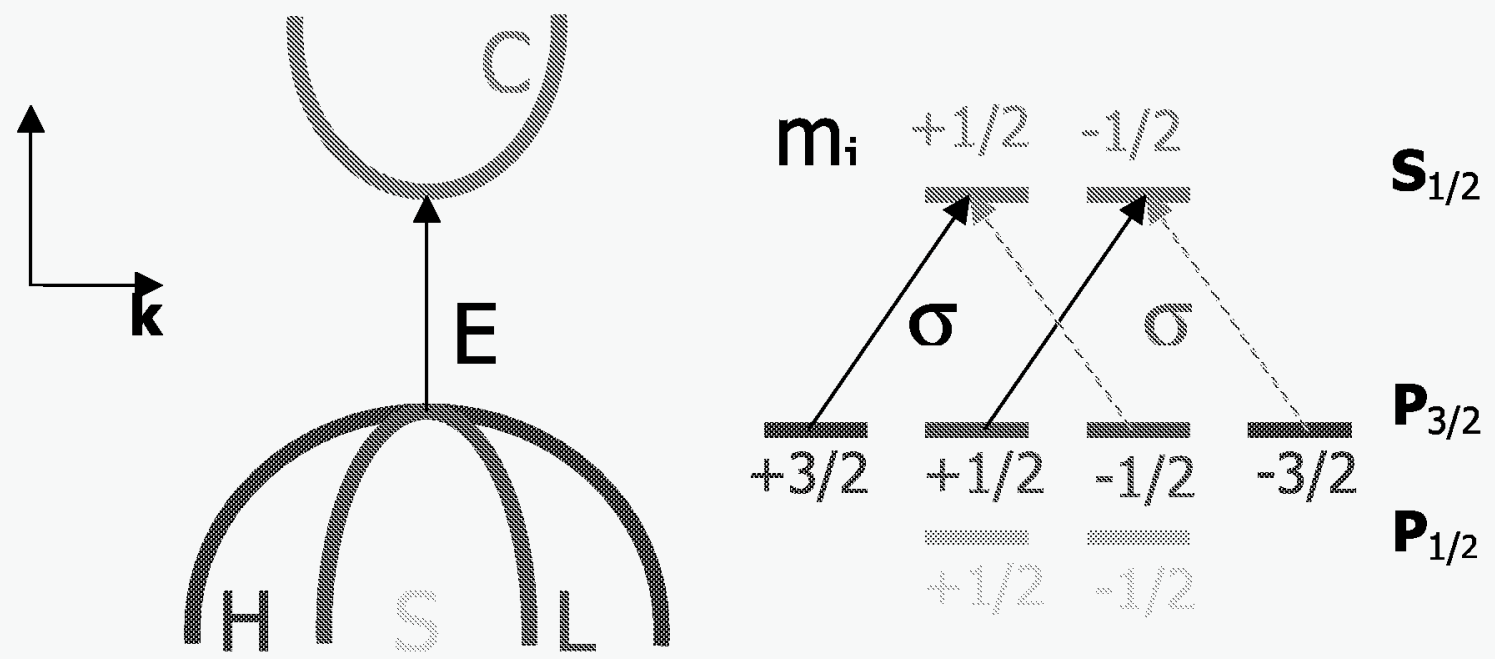

Figure 2.3. The transitions allowed in unstrained GaAs excited by circularly polarized light. Transitions take place with electron spins anti-parallel to the photon spin occur three times as much as those parallel to the photon producing a $50 \%$ spin polarization.

Alternatively, for strained GaAs, this valence band degeneracy between the heavy-hole and lighthole bands at $\mathrm{k}=0$ is removed, allowing selective excitation of a single transition, thus leading to the potential for $100 \%$ polarization of photoemitted electrons. The allowed transitions are depicted in Figure 2.4. Strain is incorporated in the GaAs layer when GaAs is epitaxially grown on a thick $\operatorname{GaAs}_{1-\mathrm{x}} \mathrm{P}_{\mathrm{x}}$ buffer layer. A larger phosphorus fraction produces a larger latticemismatch and therefore a larger strain. However, if the GaAs layer exceeds a characteristic critical thickness [10], the strain is relieved by producing misfit dislocations. This is an important point since it places a practical limit to the amount of strain in a layer. Figure 2.5 shows the critical thickness as a function of the phosphorus fraction in $\mathrm{GaAs}_{1-\mathrm{x}} \mathrm{P}_{\mathrm{x}}$. To produce a strain large enough to achieve the valence band splitting, it is necessary to use a lattice-mismatch as large as $1 \%$, i.e., the phosphorus fraction of $\mathrm{x}=0.28-0.30$, where the critical thickness is only about 10 $\mathrm{nm}$. 


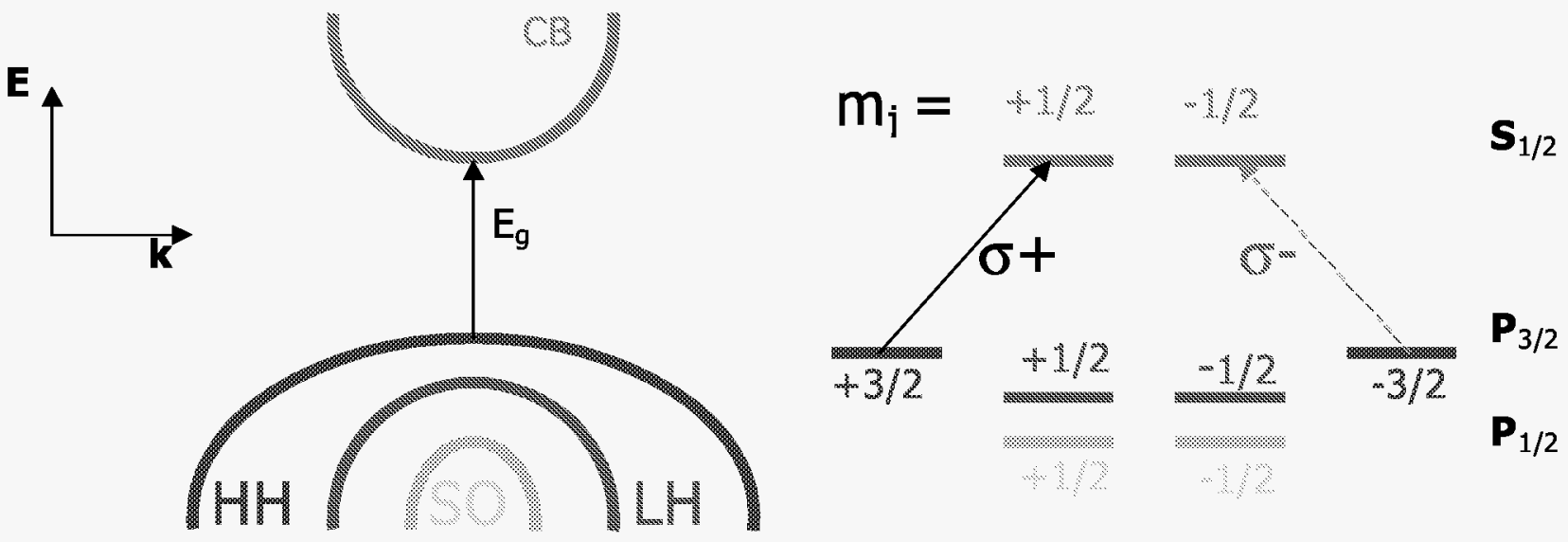

Figure 2.4. The transitions induced by circularly polarized light in strained GaAs are no longer degenerate in electron spin leading to the potential for $100 \%$ spin polarization.

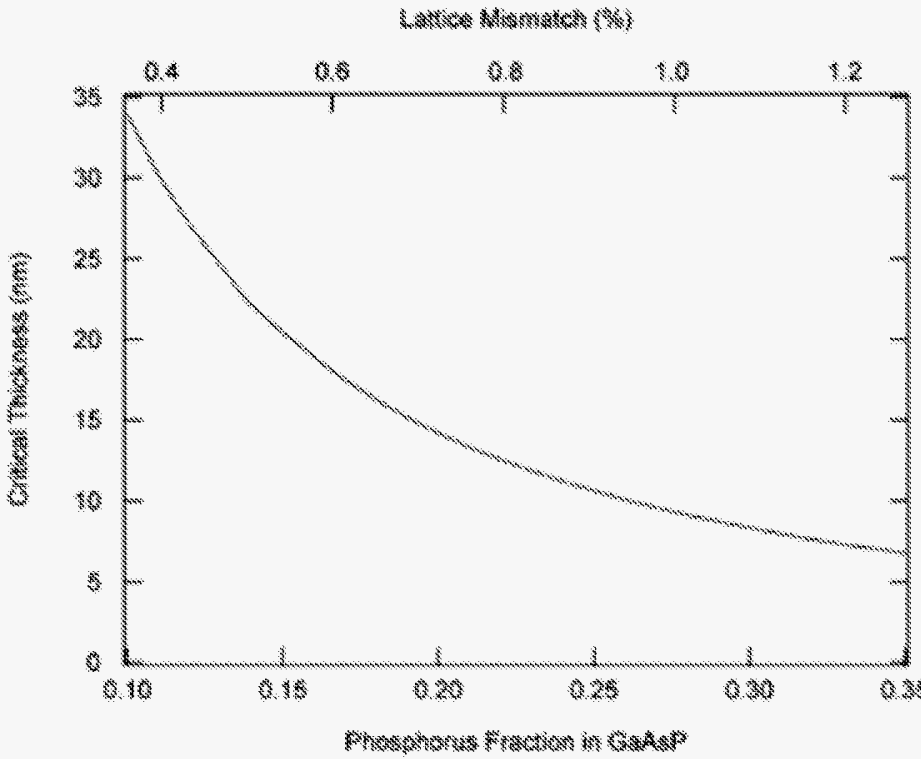

Figure 2.5.

Critical thickness of GaAs grown on GaAsP.

In practice it is not possible to attain this critical limit with a single GaAs layer, but it has been experimentally shown that the lattice strain is preserved to a thickness much beyond the critical thickness although the layer has many dislocations. This inherent thickness limitation means the crystal strain is partially relaxed and that only something less than $100 \%$ electron polarization can be achieved.

In the strained-superlattice structure investigated here (shown in Figure 2.6), the layer thickness of GaAs and GaAsP each is 3 to $5 \mathrm{~nm}$ in the superlattice, which is below the critical thickness. The strain is expected to be preserved to a higher degree than is possible in a single layer, resulting in a larger valence band energy splitting and a higher polarization. The valence band 
splitting is further increased due to the quantum-size-effect of the superlattice structure. Moreover, since the number of misfit dislocations is also reduced, conduction electron transport is improved, resulting in a higher $\mathrm{QE}$.

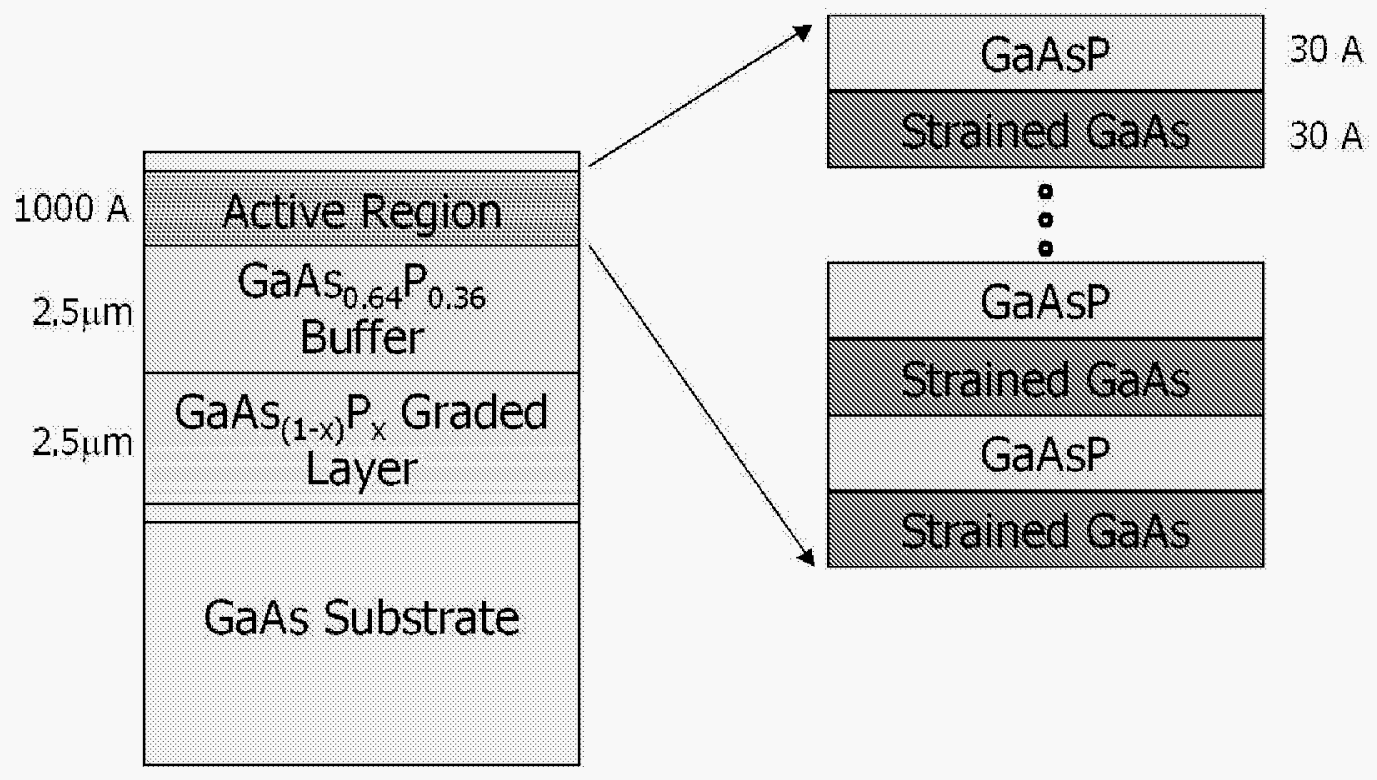

Figure 2.6. The GaAs/GaAsP superlattice structure investigated in the present program The active region consists of pairs of alternating layers of GaAs and GaAsP.

When the barrier width in the superlattice structure is reduced to several $\mathrm{nm}$, the wave functions from each well begin to overlap forming a mini-band structure, and the vertical transport becomes significant. Figure 2.7 depicts the energy band structure of the superlattice with the relative locations of the conduction mini-band, the heavy-hole and light-hole mini-bands indicated. The $\mathrm{QE}$ is expected to be higher also because the energy difference between the conduction mini-band and the heavy-hole mini-band is larger than the band gap energy of single-strained GaAs. Charge enhancement is also observed because the higher conduction mini-band boosts the energy of the conduction electrons at the surface, resulting in a reduction of the surface electron trapping that is responsible for the space charge limitation. 


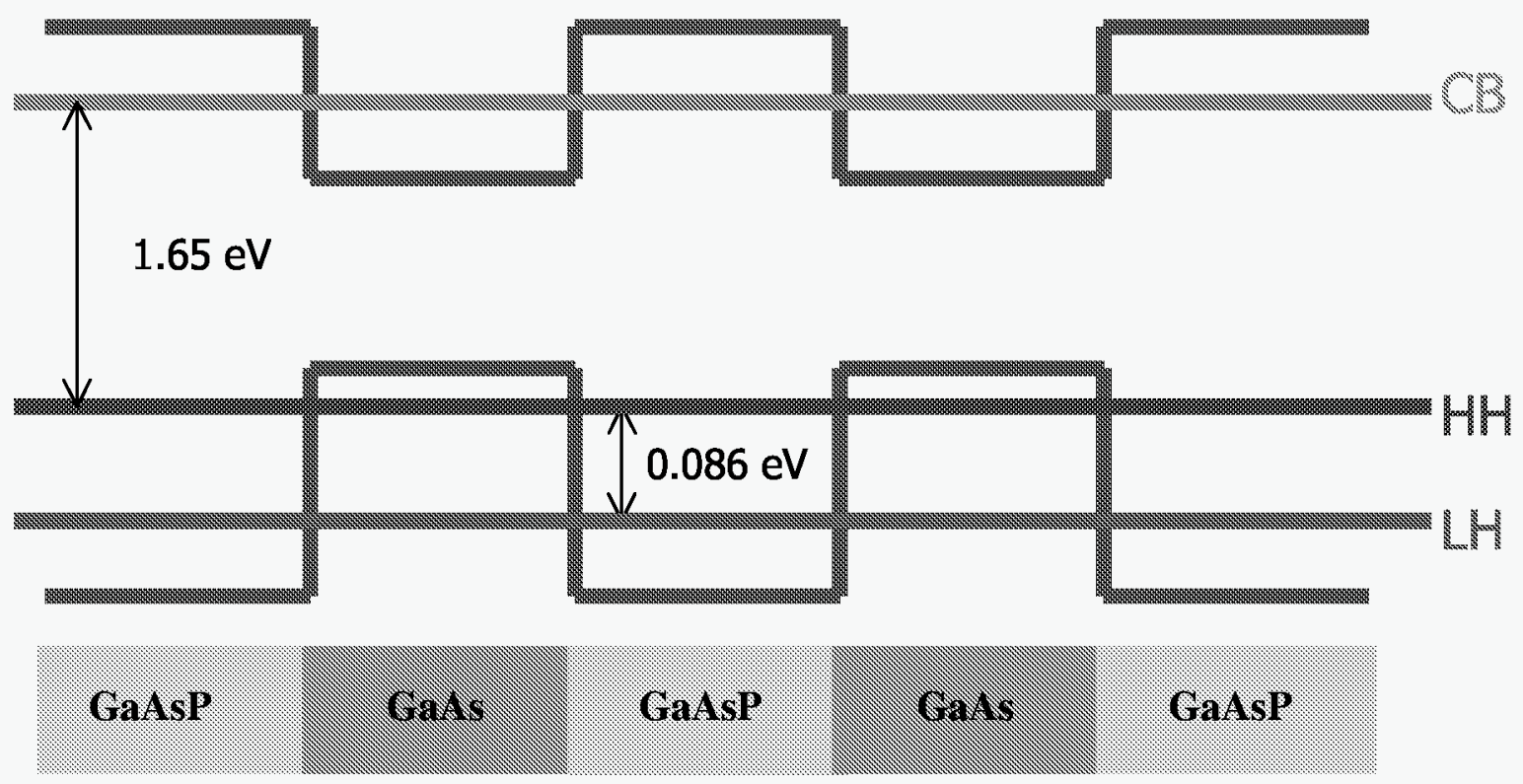

Figure 2.7. The superlattice band structure showing the splitting of the heavy and light hole valence bands.

There are three important physical parameters to be considered in the strained-superlattice structure:

1. the phosphorus fraction in $\mathrm{GaAs}_{1-\mathrm{x}} \mathrm{P}_{\mathrm{x}}$,

2. barrier (GaAsP) thickness,

3. well (GaAs) thickness.

A larger phosphorus fraction produces a larger lattice-mismatch and therefore a larger strain. However, the critical thickness must be taken into consideration since the critical thickness in the strained-superlattice structure is not well known. Two additional effects concern the superlattice structure. First, plastic relaxation of the strained layers through the introduction of extended defects can alter the performance of the photocathode. Second, even in the absence of crystalline defects the strained layers may develop lateral thickness variations during growth. Such variations can not only effect the individual layer, but also any subsequent layer during the growth. Consequently, material quality and uniformity must be examined and accounted for during the growth optimization process.

A thicker barrier increases the valence band splitting but decreases the vertical electron transport probability. The barrier thickness must be optimized for polarization and $\mathrm{QE}$.

In addition to these three parameters, the doping profile is important. Doping must be highly ptype to achieve large band-bending, thereby allowing formation of a good negative-electronaffinity surface. The doping level of the single-strained GaAs photocathodes used at SLAC is $5 \times$ $10^{18} \mathrm{~cm}^{-3}$. A recent study has shown that the surface charge effect is strongly dependent on the doping level, diminishing to zero at a doping level of $5 \times 10^{19} \mathrm{~cm}^{-3}$ [11]. However, increasing the doping level de-polarizes the electron spin. To increase the doping level while maintaining high polarization, a modulated dopant-concentration technique can be used. The strained-superlattice is doped at $5 \times 10^{17} \mathrm{~cm}^{-3}$ with a $10 \mathrm{~nm}$ GaAs cap layer doped at $5 \times 10^{19} \mathrm{~cm}^{-3}$. The low doping 
level (lower than the standard $5 \times 10^{18} \mathrm{~cm}^{-3}$ ) achieves high polarization, while the surface high doping solves the charge limit problem. Since the high doping level de-polarizes the electron spin, the thickness of this layer must be minimized.

\section{PREVIOUS TECHNOLOGY}

The cathode used in the accelerator operation at SLAC prior to this program was a high-gradientdoped strained GaAs/GaAsP [12]. The MOCVD-grown (metal organic chemical vapor deposition) cathode consisted of a strained GaAs low-doped layer (with a small admixture of $\mathrm{P}$ ) capped by a few nanometers of highly Zn-doped GaAs. When the 2-inch cathode wafer was received, it was anodized at $2.5 \mathrm{~V}$ to form a 3 -nm oxide protecting layer, and then waxed on glass and cut to the required circular shape. When the cathode is ready for installation, the glass is removed, and the cathode is degreased in boiling trichloroethane. After degreasing, the surface oxide of the cathode is stripped by ammonium hydroxide, and the cathode is immediately transferred into a vacuum loading chamber. The cathode is heat-cleaned at $600^{\circ} \mathrm{C}$ for one hour and activated by $\mathrm{Cs} / \mathrm{NF}_{3}$ co-deposition. In our standard procedure, heat cleaning and activation are done twice before the cathode is transferred into the polarized electron gun.

Although the standard procedures for cathode preparation had yielded satisfactory results, weaknesses have prevented those cathodes from achieving the ultimate desired performance.

MOCVD: The base pressure in MOCVD growth chambers is usually in low-vacuum range. Compared with ultra-high-vacuum techniques like MBE, the growth environment of MOCVD may not be as clean. Furthermore, because chemical reactions are needed on wafer surfaces during growth, MOCVD requires higher growth temperature, and its growth mechanism is complicated. Due to the nature of the MOCVD process, it may be difficult to grow thin films of the highest quality.

Zn dopant: The diffusion coefficient of $\mathrm{Zn}$ in $\mathrm{GaAs}$ is high at the heat-cleaning temperature in our preparation procedure $\left(600^{\circ} \mathrm{C}\right)$. Because of the high diffusion coefficient, the heat-cleaning capability of $\mathrm{Zn}$-doped cathodes is very limited. In high-gradient-doped cathodes, the highly doped surface layer is responsible for removing the surface-charge-limit effect in high photocurrent operations [12], and dopant loss is very undesirable.

The SIMS (Secondary Ion Mass Spectroscopy) analysis shown in Fig. 3.1 confirms the Zn-dopant loss after cathodes receive heat-cleaning treatments. Before the cathode is heat-cleaned, the $\mathrm{Zn}$ dopant concentration is $5 \times 10^{19} \mathrm{~cm}^{-3}$ as specified [12]. After 5 hours of heat-cleaning at $600^{\circ} \mathrm{C}$, the $\mathrm{Zn}$-doping level at surface drops significantly. A test on a Zn-doped high-gradient-doped cathode indicates that the cathode starts to show the surface-charge-limit effect after three hours of heatcleaning at $600^{\circ} \mathrm{C}$. 


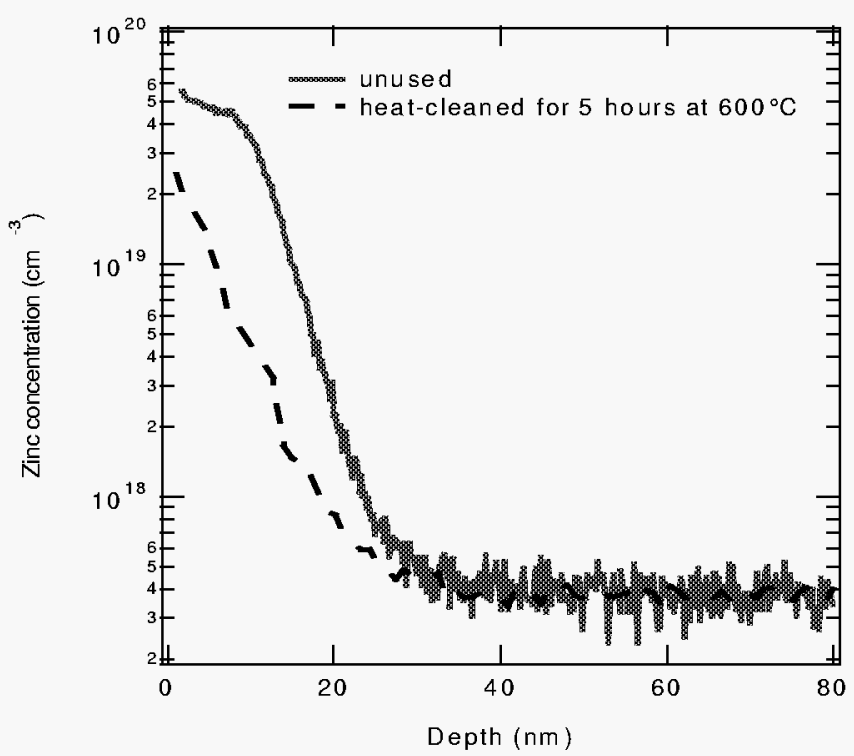

Figure 3.1

SIMS analysis for the Zn-doped cathode before and after heat cleanings.

\section{PHASE I RESULTS}

The main objective of the Phase I program was the realization of a high performance photocathode to serve as a polarized electron source. The GaAs/GaAsP superlattice structure shown in Figure 2.6 was chosen as the candidate material for this purpose. With one such superlattice (SVTA 3682) whose active region consisted of 16 pairs of GaAs and GaAsP layers, we were able to attain an $86 \%$ degree of spin polarization and a quantum efficiency of more than $0.2 \%$. These results, shown in Figure 4.1, represent a significant and encouraging achievement. It was the highest degree of polarization ever achieved with a photocathode at SLAC. This success validated our technical approach and was the result of detailed physics modeling and well-controlled material fabrication.

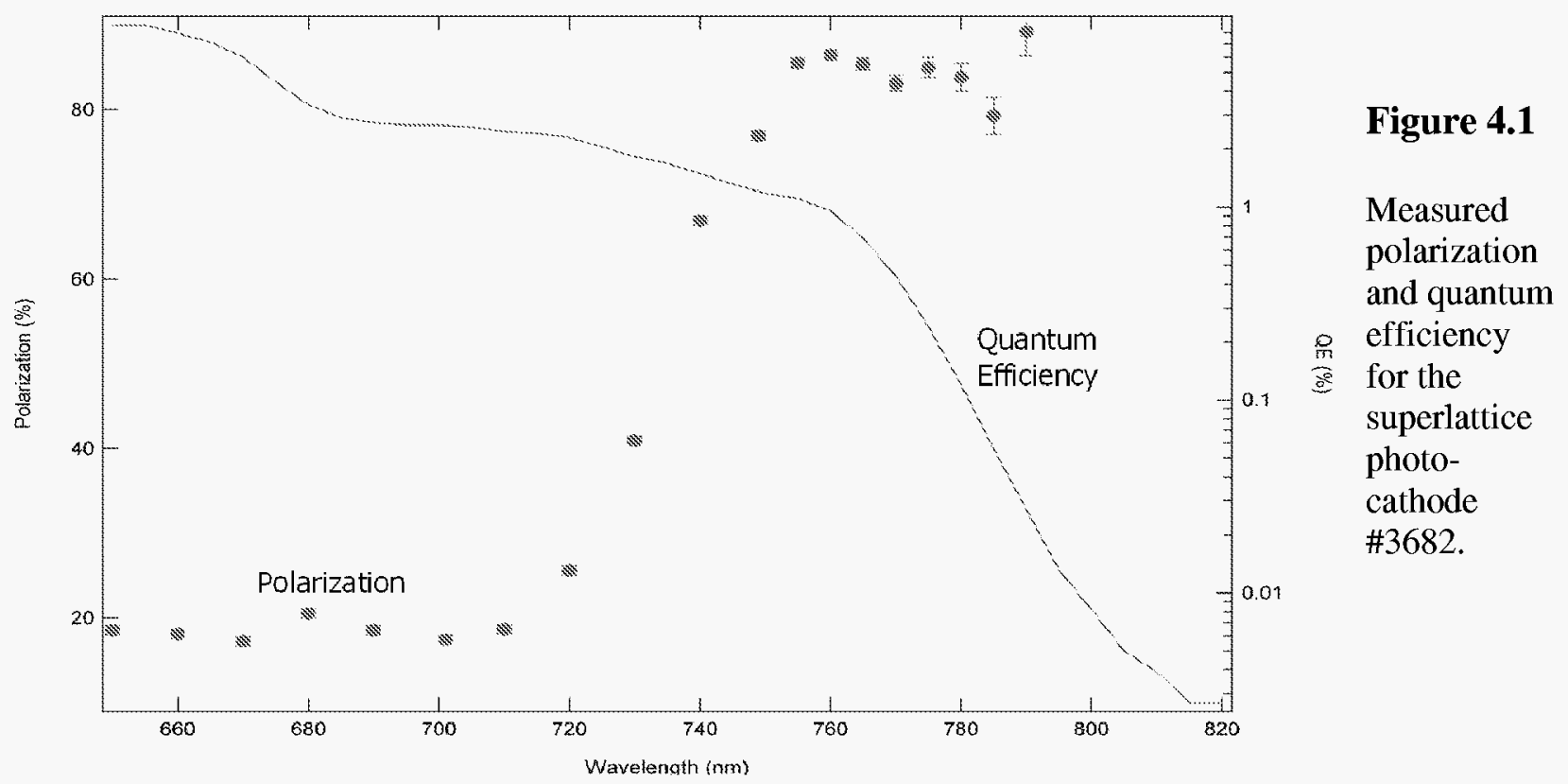


Use of strained semiconductor enhances the polarization of the electron emission. To achieve larger currents, the volume of the active layer must be increased (greater thickness). However, when the strained material reaches a critical thickness, it relaxes and loses its strained characteristics. As a practical workaround for this problem, a superlattice is grown, consisting of alternating strained and non-strained layers. Each strained layer is thin enough to prevent relaxation. A theoretical model employing the $\mathrm{k} \bullet \mathrm{p}$ transfer matrix was developed to help predict the optimal design of the superlattice structure. Figure 4.2 shows the results of the band calculations for one such structure. The simulation predicts an effective band gap energy of 1.65 $\mathrm{eV}$ and an LH-HH splitting of $86 \mathrm{meV}$ (see Figure 2.7).
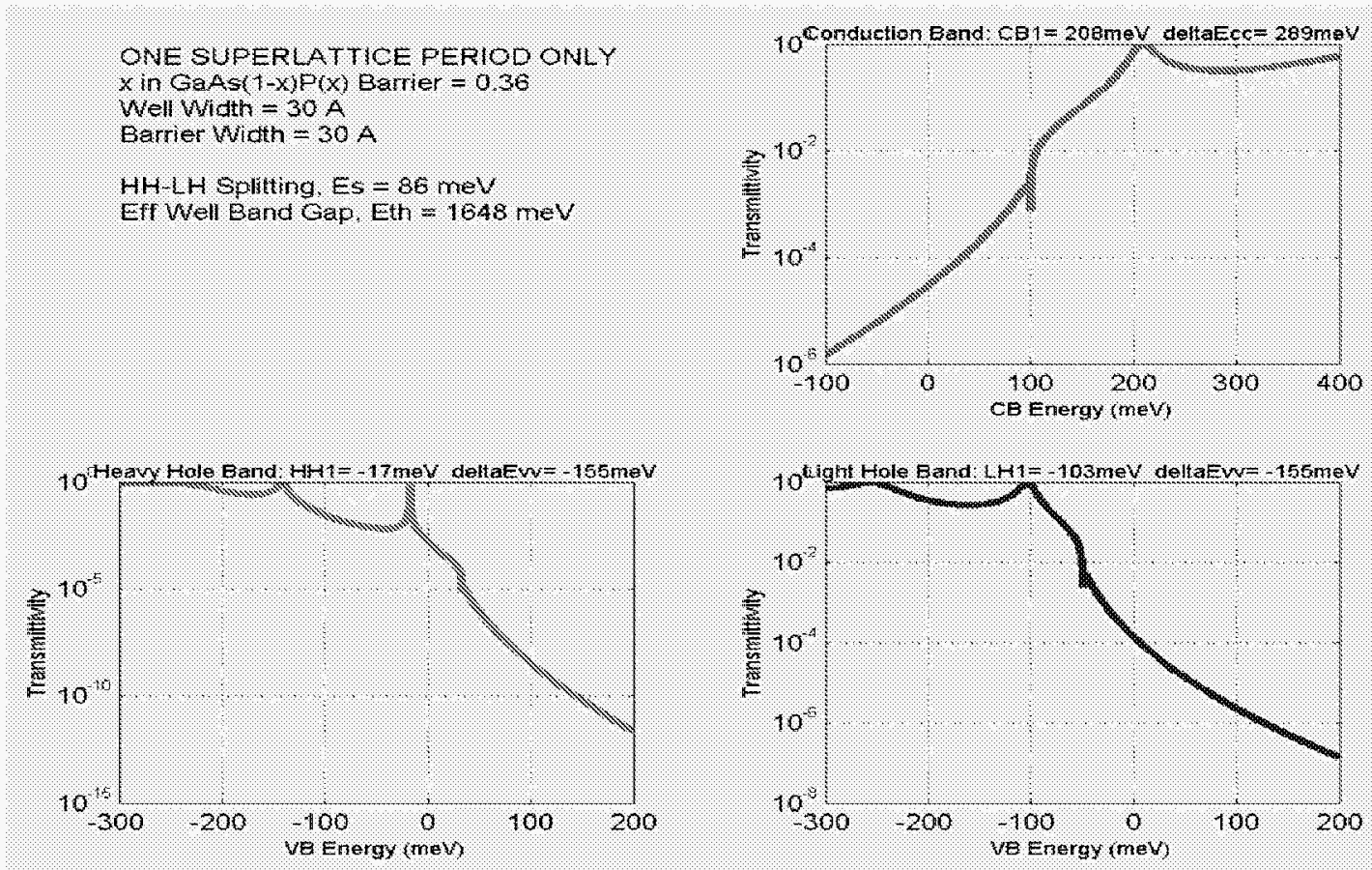

Figure 4.2. Simulation of sample \#3682 utilizing $30 \AA$ thick well and barrier layers.

Although several series of the strained superlattice devices were grown and fabricated for this Phase-I project, the best result is obtained (\#3682) using $30 \AA$ thick superlattice layers (as shown previously in Figure 4.1) as in the simulation of Figure 4.2. Room temperature photoluminescence of the grown structure yielded emission at $1.65 \mathrm{eV}$, which was the predicted effective band gap energy from the superlattice (Figure 4.3). X-ray diffraction analysis of the sample matched the simulated data (Figure 4.4) very closely indicating high structural perfection. These results support the validity of the theory and the actual fabrication of the designed structure. For photocathodes, test samples were cut into smaller pieces, discs approximately $2 \mathrm{~cm}$ in diameter, and surface treatment was applied. The photocathode was then loaded into the SLAC Cathode Test System. The photocathode was heat-cleaned at $600^{\circ} \mathrm{C}$, cooled down to room temperature, and then activated using cesium and nitrogen-trifluoride. The quantum efficiency and polarization were measured using a tungsten lamp and a monochrometer as a light source. 
\#3682 Photoluminescence

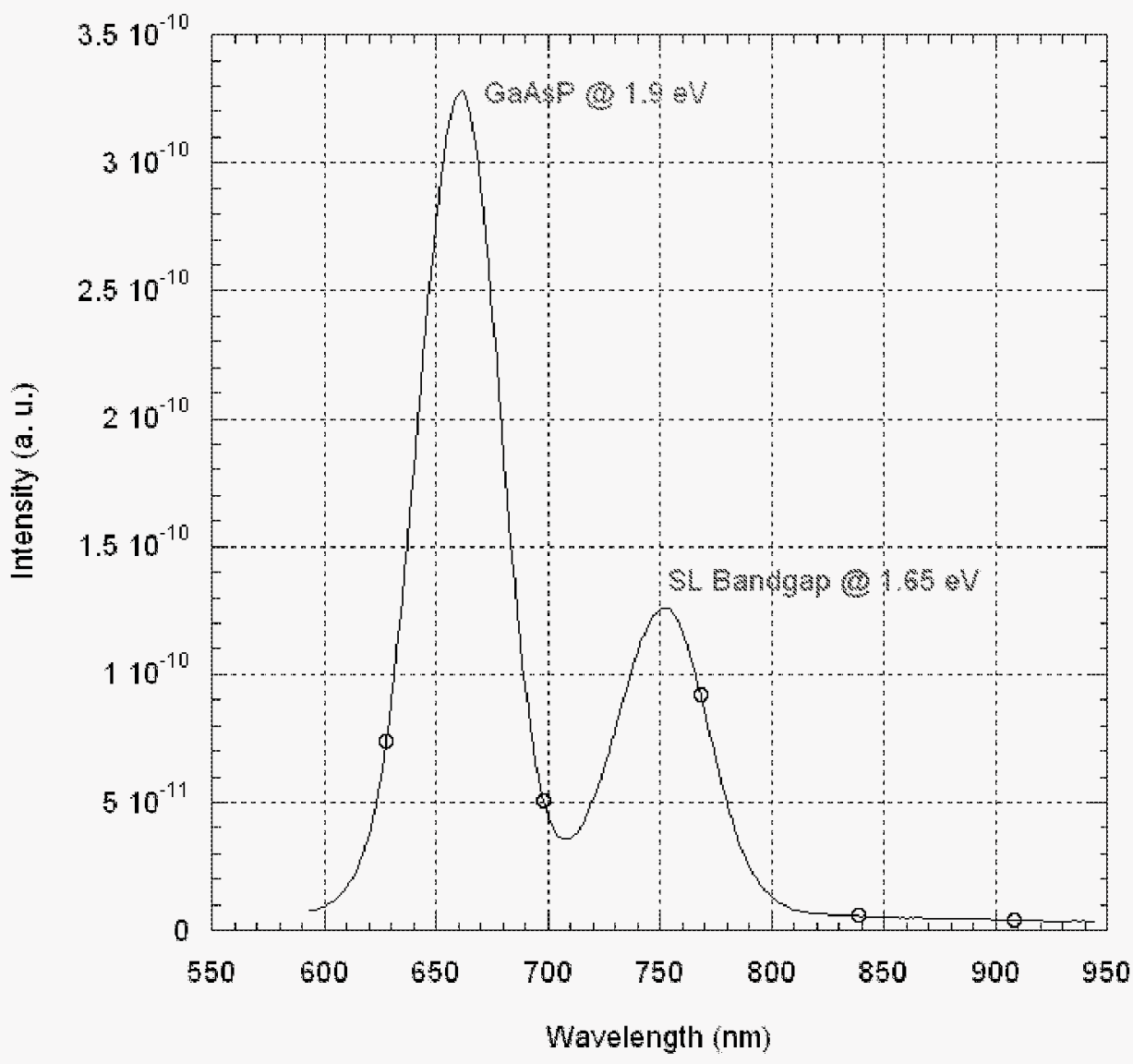

Figure 4.3.

Photoluminescence of the \#3682 sample at room temperature.

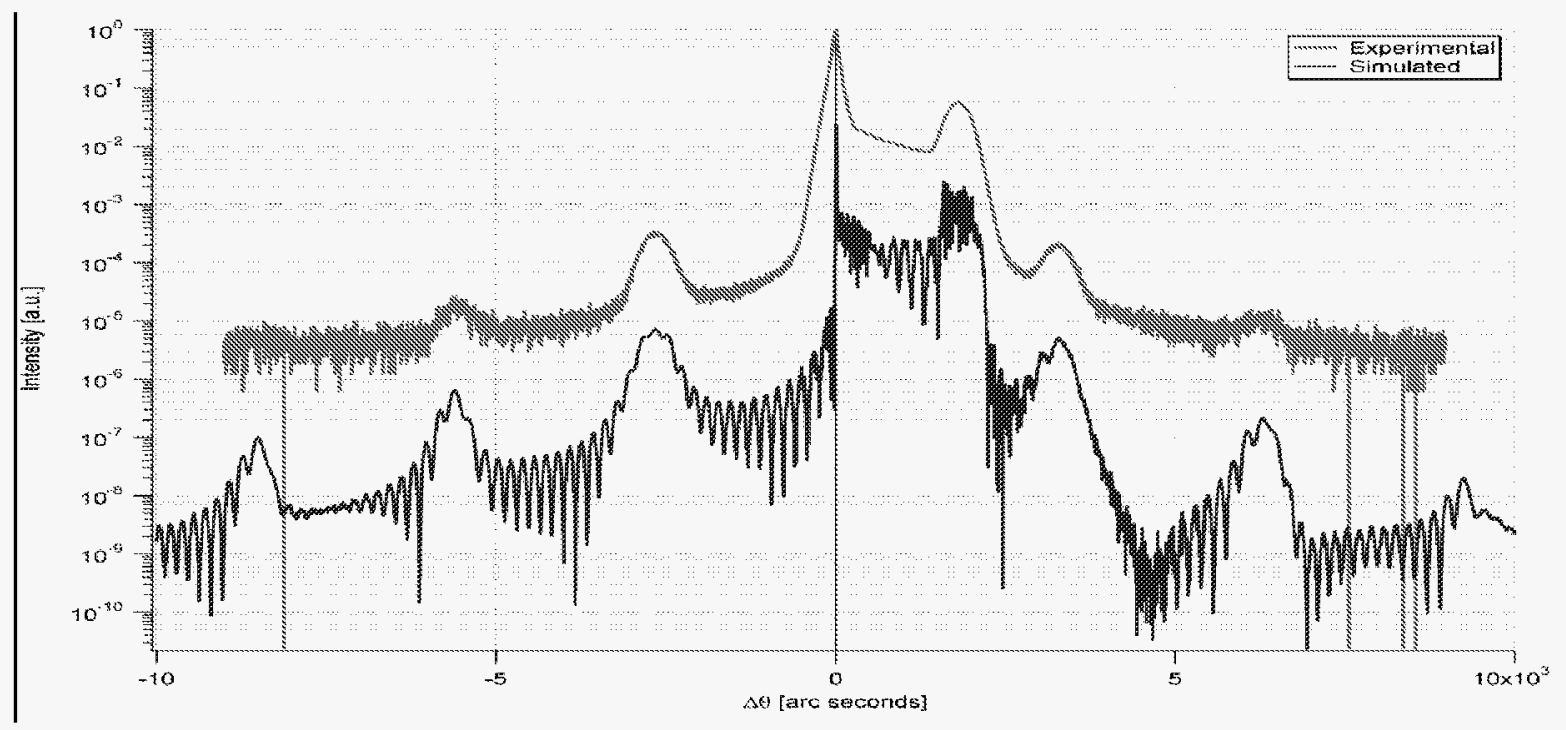

Figure 4.4. Measured and simulated $x$-ray diffraction data from the \#3682 structure, showing excellent agreement of all the major diffraction peaks.

Figure 4.5 shows the polarization and quantum efficiency as a function of wavelength for a number of photocathode samples. For wavelengths corresponding to the superlattice effective 
bandgap energy and longer, the electron polarization reaches levels over $80 \%$ with a peak at $86 \%$ (for the sample \#3682 as shown in Figure 4.1). This was the highest polarization value measured at SLAC at the time. The quantum efficiency at $760 \mathrm{~nm}$, where the polarization is maximum, is $1 \%$. This value is at least a factor of three higher than for the SLAC standard strained photocathodes. In summary, the Phase I results proved to advance the state-of-the-art for photocathodes.
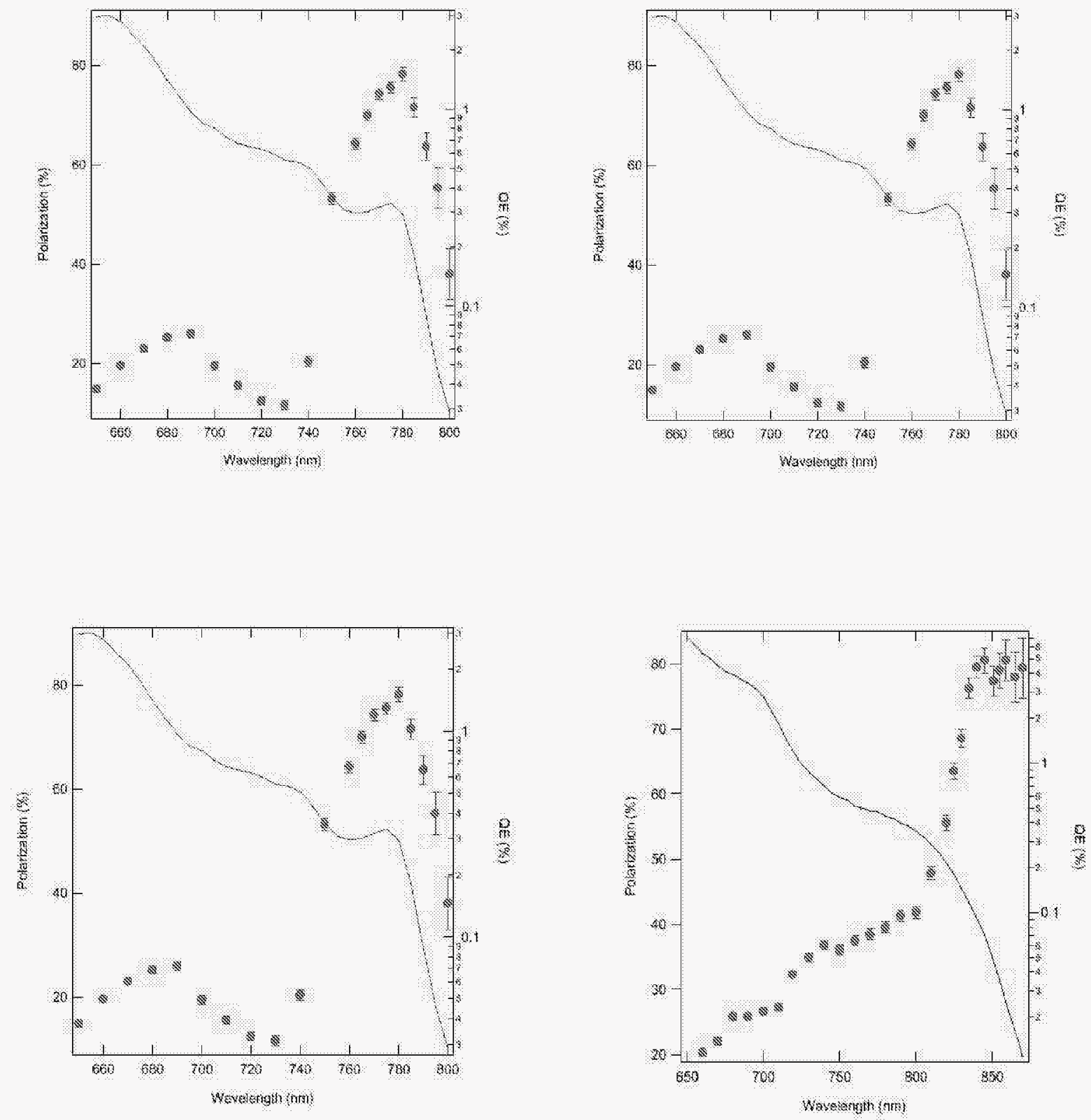

Figure 4.5. Polarization and quantum efficiency measurements obtained for various superlattice samples grown during the Phase I program. All attained polarization as high as $80 \%$. 


\section{METHODS AND APPARATUSES}

\section{Molecular Beam Epitaxy}

Fabrication of the superlattice structure was carried out by molecular beam epitaxy (MBE) technique. MBE is an extremely versatile method for growing semiconductor compounds and multilayer structures. In MBE, fluxes of various elements converge on a heated wafer forming the desired compound in a controlled environment (Fig. 5.1). Each elemental beam flux is generated by heating the material in special ovens (called effusion cells) in an ultrahigh vacuum chamber (base pressure $<5 \times 10^{-11}$ torr). To obtain high quality material the MBE process parameters must be precisely controlled. The fluxes of various elemental beams and the substrate growth temperature have to be maintained at extremely stable conditions

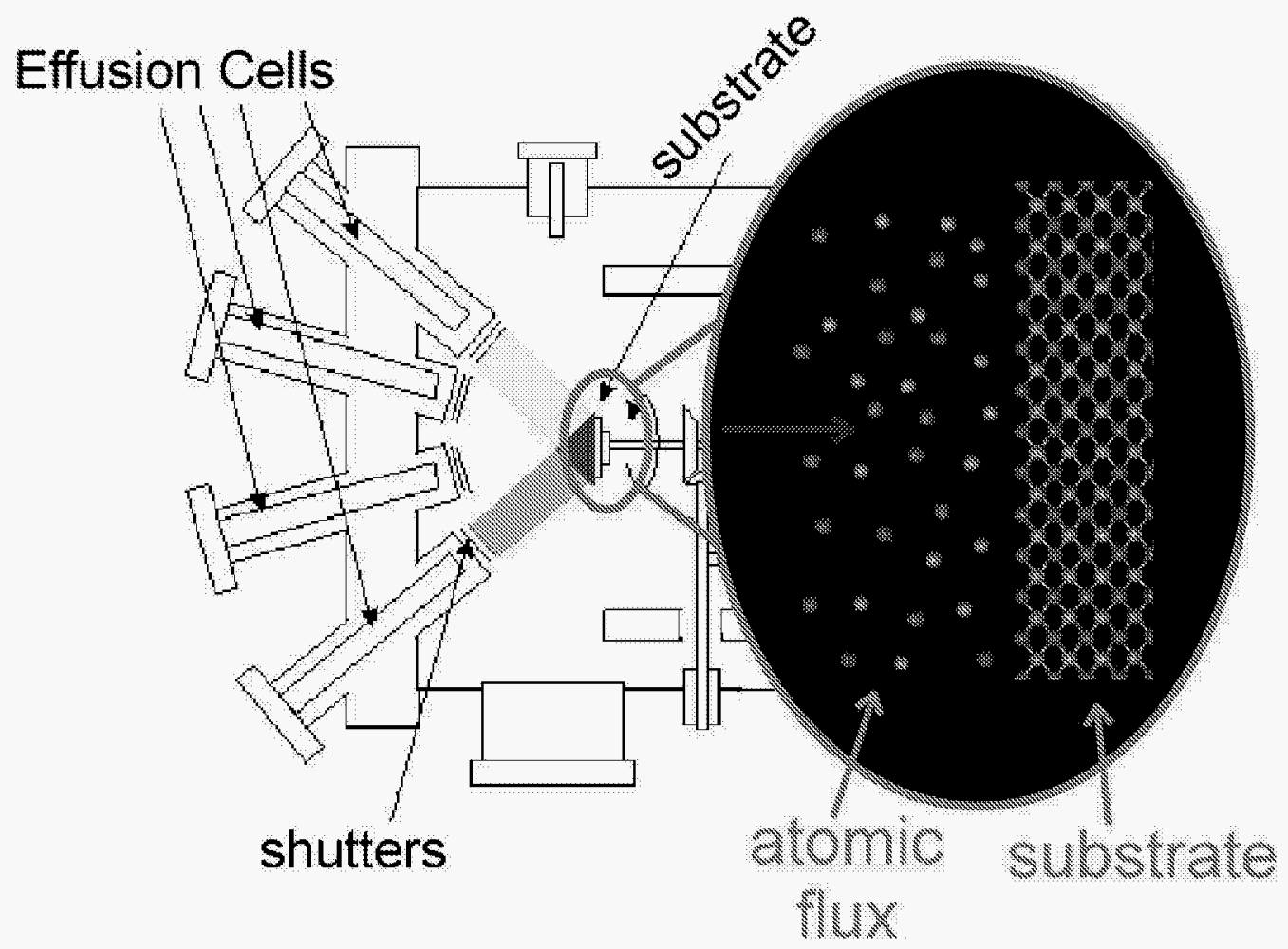

Figure 5.1. Illustration of the molecular beam epitaxy (MBE) technique showing the ultra high vacuum growth chamber with individual high purity elemental sources.

A 0.2- $\mu$ m-thick $p$-type GaAs buffer layer was grown on a (100) $n$-type GaAs substrate. In order to produce a strain-relieved $\mathrm{GaAs}_{1-x} \mathrm{P}_{x}$ layer on GaAs, a $2.5-\mu \mathrm{m}$-thick $\mathrm{GaAs}_{1-y} \mathrm{P}_{y}$ layer was grown with an increasing phosphorus fraction from $y=0$ to $x$ to accommodate the lattice mismatch, followed by an additional $2.5-\mu \mathrm{m}$ thick $\mathrm{GaAs}_{1-x} \mathrm{P}_{x}$ layer with a fixed phosphorus fraction. The superlattice structure was then grown on this layer. The phosphorus fraction in the superlattice barrier $\mathrm{GaAs}_{1-x} \mathrm{P}_{x}$ was the same as in the buffer $\mathrm{GaA}_{s 1-x} \mathrm{P}_{x}$ layer so that the superlattice GaAs well layers were fully strained while the superlattice $\operatorname{GaAs}_{1-x} \mathrm{P}_{x}$ barrier layers were fully relaxed. A short growth interruption was adopted at each superlattice interface for switching the 
phosphorus flux. The superlattice layer was doped with beryllium to a value of $5 \times 10^{17} \mathrm{~cm}^{-3}$. On top of the superlattice layer, a 5-10 nm-thick GaAs surface layer was grown with a doping concentration of $5 \times 10^{19} \mathrm{~cm}^{-3}$.

\section{Cathode Test System}

Polarization and quantum efficiency (QE) are measured in the SLAC Cathode Test System (CTS) shown schematically in Figure 5.2. The CTS is an ultra-high vacuum system pumped by a combination of ion and non-evaporable getter (NEG) pumps. The system is equipped with a load-lock chamber through which samples can be introduced without venting the system vacuum. Polarization measurements are accomplished by an electron transport column, an electrostatic $90^{\circ}$ spin-rotator and a $20 \mathrm{keV}$ Mott polarimeter. The photon helicity is controlled by a linearpolarizer/liquid-crystal retarder combination.

Prior to installation in the system, the sample is degreased in a boiling solution of trichloroethane. After the protective oxide layer is removed in ammonium hydroxide, the sample is rinsed in distilled water and methanol. The cathode activation method used to obtain a negative-electronaffinity (NEA) surface consists of heat cleaning to $600^{\circ} \mathrm{C}$ for 1 hour, cool-down for an hour, followed by application of cesium until the photo-yield peaks, and then cesium and nitrogentrifluoride co-deposition until the photo-yield is again maximized. The heat-cleaning temperature is monitored via an infrared pyrometer. The cathode is activated while monitoring the photoyield with a white light and a $670 \mathrm{~nm}$ diode laser. Once a red response is observed from the diode laser, the white light is turned off and the diode laser is used to complete the activation. The absolute $\mathrm{QE}$ is measured using the diode laser at a photon wavelength of $670 \mathrm{~nm}$. A tungsten lamp and a monochrometer are used to measure the relative $\mathrm{QE}$ as a function of photon wavelength, and these measurements are then normalized to the diode laser measurement at 670 $\mathrm{nm}$.

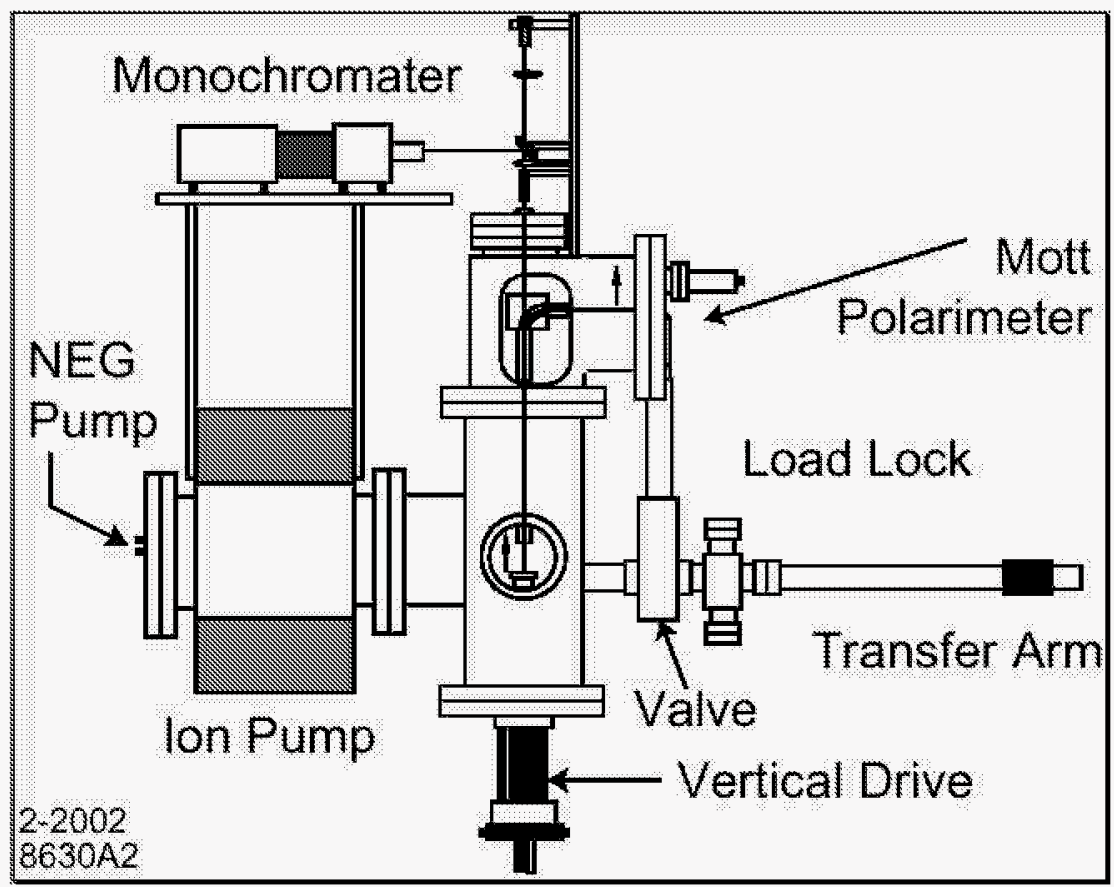

Figure 5.2

A schematic view of the SLAC cathode test system (CTS). 


\section{SLAC Gun Test Laboratory}

The SLAC Gun Test Laboratory (GTL) is used to study the production of intense, highlypolarized electron beams required for the accelerator. The laboratory, shown schematically in Figure 5.3, is a replica of the first few meters of the SLAC injector beamline. The GTL beamline consists of an ultra-high-vacuum, high-voltage electrostatic gun, a load-lock chamber for cathode transfer and activation (not shown in Figure 5.3), and a beamline with magnetic components for electron beam transport and steering. An electrically isolated, optically coupled nanoammeter is used to measure the average photoemission current. The cathode is biased at $-120 \mathrm{kV}$ and maintained at a temperature of $0 \pm 2^{\circ} \mathrm{C}$ by circulating cold nitrogen gas. With the cathode biased

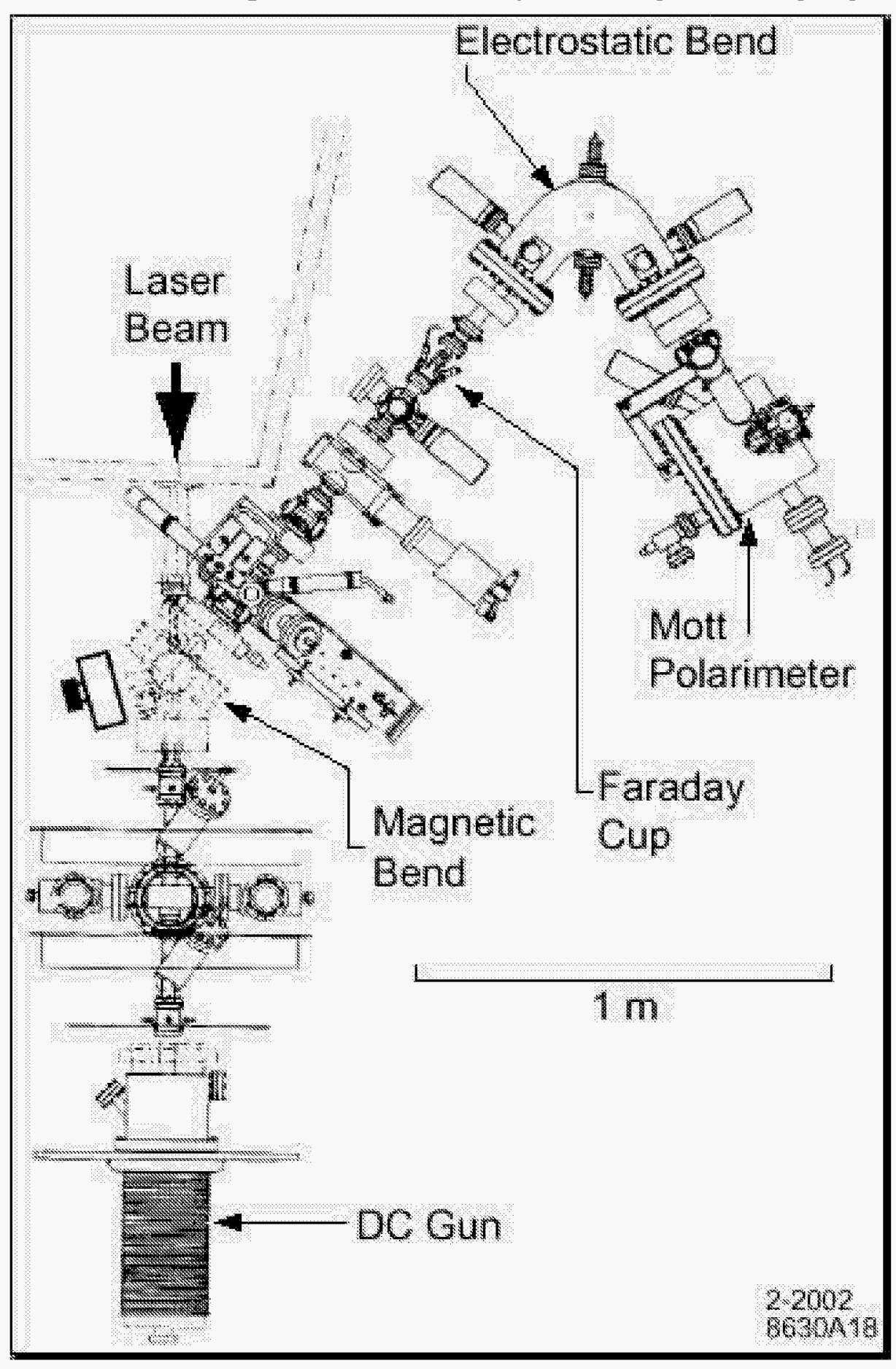

at $-120 \mathrm{kV}$, the field gradient at the cathode is $1.8 \mathrm{MV} / \mathrm{m}$, and the gun is capable of extracting a spacecharge-limited current of $15 \mathrm{~A}$ from the 20 $\mathrm{mm}$ diameter cathode. A fast Faraday cup is used to measure the temporal profile of the beam with a time resolution of $0.5 \mathrm{~ns}$. The Faraday cup has a $2 \mathrm{~mm}$ diameter hole through which a fraction of the beam is transported to the Mott chamber for polarization measurements. The vacuum in the gun is maintained at about $1 \times 10^{-11}$ Torr by means of ion and nonevaporative getter pumping.

\section{Figure 5.3}

A schematic view of the SLAC gun test laboratory (GTL).

The cathode is activated in the GTL load-lock chamber. 
The cathode preparation and activation procedures are similar to those used for the CTS. The heat-cleaning temperature is measured at the back surface of the cathode holder using a thermocouple. The temperature at the cathode surface is estimated to be about $600^{\circ} \mathrm{C}$ when the thermocouple temperature is $800^{\circ} \mathrm{C}$. Cathodes are typically heat-cleaned and activated twice before being moved into the gun. Once the cathode is moved into the gun, the cathode QE can be restored by application of cesium without further heat-cleaning or exposure to nitrogen trifluoride. The cathode lifetime is continuously monitored using a $750 \mathrm{~nm}$ diode laser running at $1.5 \mathrm{~Hz}$ and a lock-in amplifier to detect the modulated current of the nanoammeter.

\section{INITIAL COMPARISON WITH PREVIOUS PHOTOCATHODES}

\section{Strain relaxation in single strained layer:}

The high-gradient-doped cathode had a 90nm thick strained GaAsP as its active layer. The strain in the active layer is responsible for the polarization of photoelectrons, and strain relaxation in the active layer will lower the polarization.

A strained layer starts relaxing when its thickness exceeds the critical thickness. It relaxes completely when its thickness exceeds the practical thickness. It is partially relaxed when its thickness is between the critical thickness and the practical thickness. In the case of GaAs or GaAsP, the critical thickness is about $10 \mathrm{~nm}$, and the practical thickness is about $100 \mathrm{~nm}$. Table I shows the comparison of the performance between two cathodes. Both cathodes are highgradient-doped strained GaAs/GaAsP. The only difference between the two cathodes is the thickness of their active layers. The polarization of the cathode MO5-6007 is considerably lower due to the strain relaxation in its active layer.

Table I. Comparison of photocathode samples with high strain

\begin{tabular}{ccc}
\hline Cathode No. & $\begin{array}{c}\text { Active Layer } \\
\text { Thickness (nm) }\end{array}$ & $\begin{array}{c}\text { Polarization } \\
(\%)\end{array}$ \\
\hline MO5-5868 & 90 & 82 \\
MO5-6007 & 170 & 70 \\
\hline
\end{tabular}

To address the weaknesses in the standard cathode growth and preparation procedure, a few improvements were made.

MBE and Be doping: The photocathode growth technique had been switched from MOCVD with $\mathrm{Zn}$ doping to $\mathrm{MBE}$ with Be doping. The ultra-high vacuum of MBE growth ensured a clean growth environment. MBE growth usually requires a lower growth temperature and has a simpler growth mechanism. All these advantages make the growth of high-quality layers more realizable. Be has much lower diffusion coefficients than $\mathrm{Zn}$, and Be-doped cathodes will have better heat-cleaning capability. 
Figure 6.1 shows the performance of the two cathodes from wafers SVT-3982 (MBE-grown Bedoped) and MO5-5868 (MOCVD-grown Zn-doped), tested in Cathode Test Laboratory (CTL) at SLAC. Both cathodes are high-gradient-doped strained GaAsP with the same structure. The result shows that the performance of MBE-grown SVT-3982 is better than MOCVD-grown SVT5868.

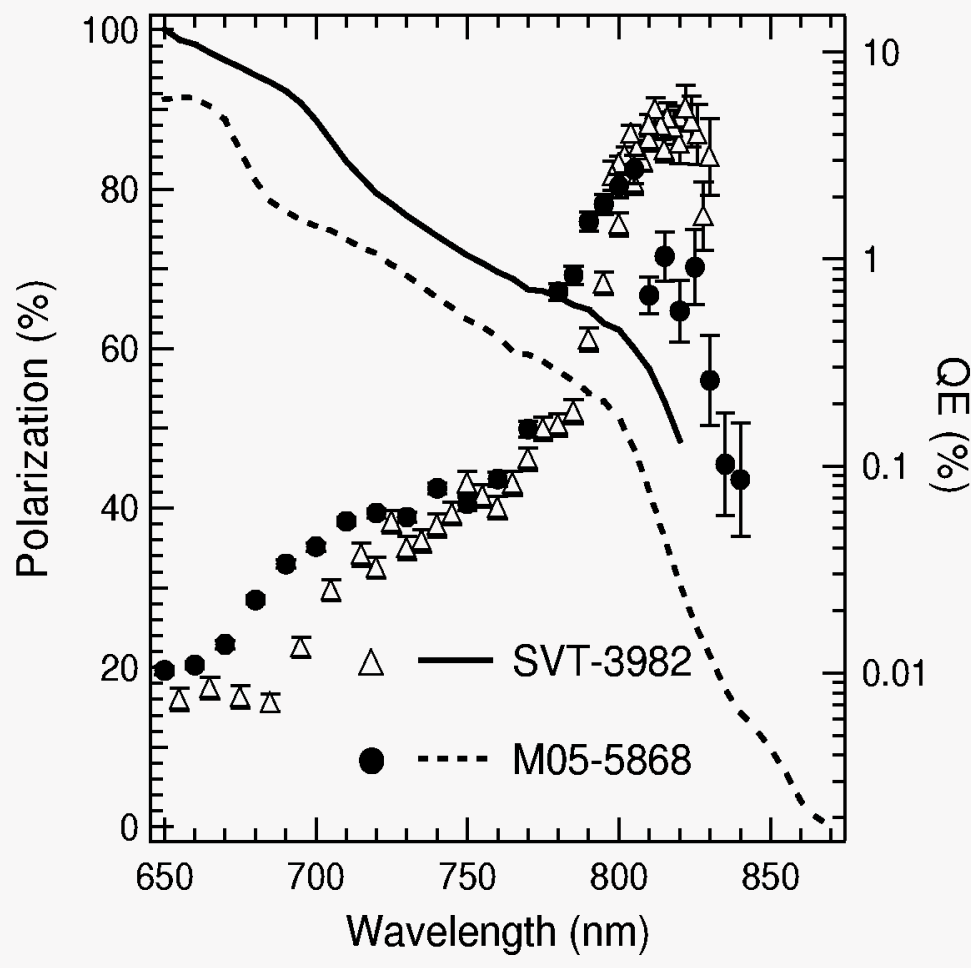

Figure 6.1.

The performance comparison between MBE-grown Be-doped (SVT-3982) and MOCVD-grown Zndoped (MO5-5868) cathodes.

Atomic hydrogen cleaning: Another method to lower the heat-cleaning temperature was to use atomic hydrogen cleaning. The idea is to use atomic hydrogen generated by a RF dissociator to react with and remove oxide and carbon contamination from cathode surface at a lower temperature. Figure 6.2 shows the results from the atomic hydrogen cleaning system in CTL. The reference cathode is $\mathrm{GaAs}$, stripped of its oxide layer by $\mathrm{NH}_{4} \mathrm{OH}$, heat-cleaned, and activated by $\mathrm{Cs} / \mathrm{NF}_{3}$ co-deposition. The result shows that the $\mathrm{QE}$ increases with increasing heat-cleaning temperature, which indicates that the thin oxide layer on the cathode surface from the short air exposure is gradually removed as the temperature increases. The GaAs test cathode is treated as indicated in the figure and then activated by $\mathrm{Cs} / \mathrm{NF}_{3}$ co-deposition. Because the test cathode is not stripped by $\mathrm{NH}_{4} \mathrm{OH}$, the cathode surface starts with a thick native oxide layer. As the data indicates, repetitive heat-cleanings at $450^{\circ} \mathrm{C}$ cannot remove the oxide layer effectively, and thus the $\mathrm{QE}$ is very low. After one hour of atomic hydrogen cleaning, the $\mathrm{QE}$ is greatly improved and is similar to that from the reference cathode heat-cleaned at $500^{\circ} \mathrm{C}$. Later studies have demonstrated that it is possible to produce cathode with a QE higher than 14\% from unstripped GaAs cathode by atomic-hydrogen cleaning (not shown in the figure). However, these studies also indicate that excessive atomic hydrogen cleaning yields GaAs cathode surfaces with low QE. 


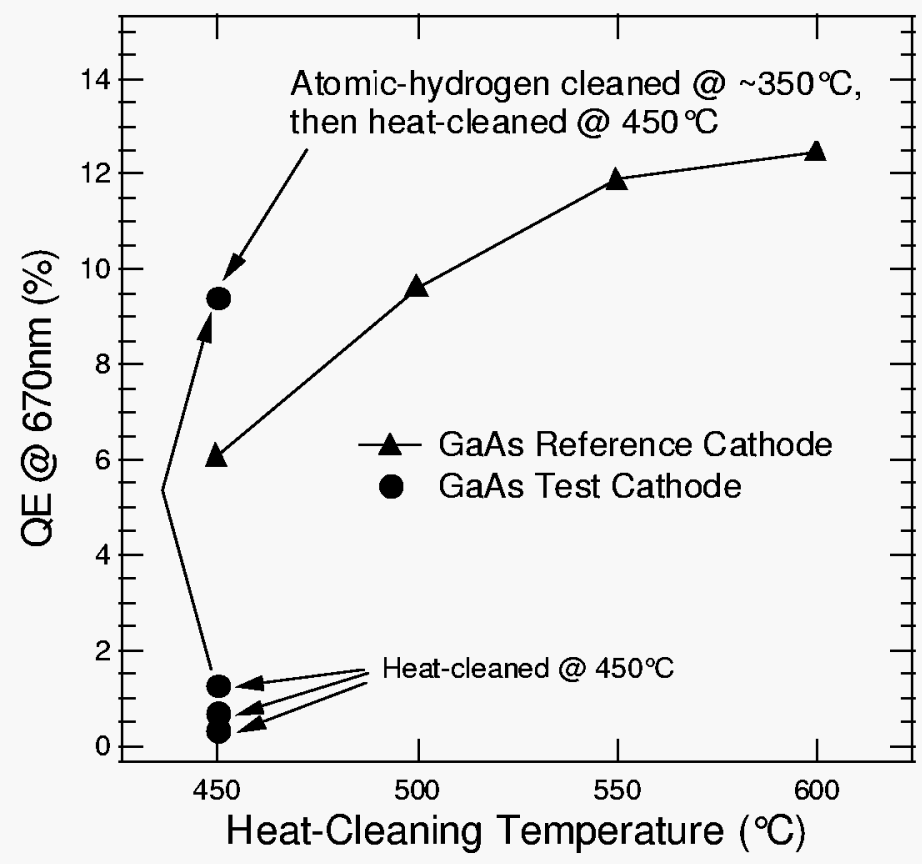

\section{Figure 6.2.}

Results from the application of atomic-hydrogen cleaning.

Superlattice photocathodes: The superlattice structure is employed to preserve strain in the active layer of photocathodes. The basis is to grow strained layers sandwiched between unstrained layers, where the thickness of each strained layer is less than the critical thickness.

1. A band structure calculation was performed to determine the proper structure parameters to grow superlattice cathodes. The transfer matrix method [13] ws used for the calculation. Fig. 4 shows typical results of effective band gap and heavy-hole-light-hole (HH-LH) splitting from the calculation. In this figure, the barrier width is fixed at $50 \mathrm{~nm}$, while the well width and the phosphorus fraction were allowed to change. Because the cathode QE is related to the band gap, and the polarization is related to HH-LH splitting, the result from calculation can be used to predict roughly how the cathode $\mathrm{QE}$ and the polarization will change when the structure parameters are varied.

2. Photoluminescence measurements were performed on cathode wafers to check the cathode band structure. This provided a way to check the accuracy of the band structure calculation. Furthermore, it also provided feedback on the uniformity of cathode wafers if the photoluminescence measurements were performed across the entire surface.

3. X-ray diffraction was performed to characterize the cathode structure when cathode wafers were received after growth. By studying x-ray diffraction patterns, structure parameters can be determined, including layer thickness, composition, and strain. 


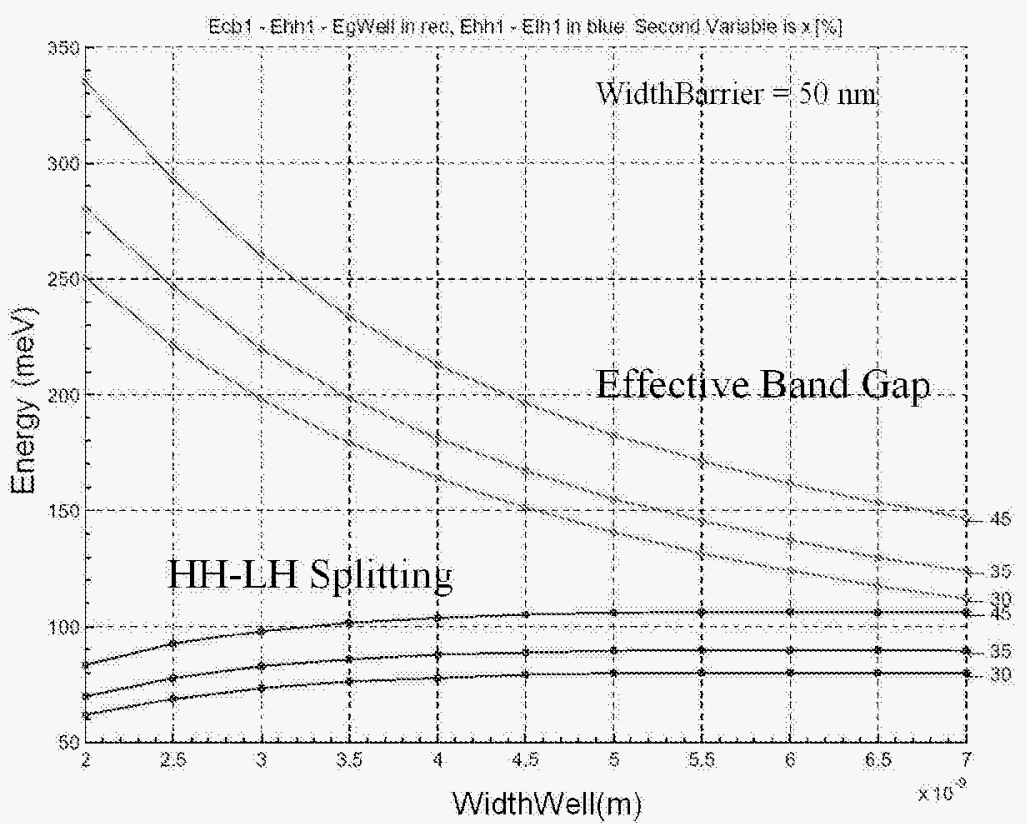

Figure 6.3.

Typical results from band structure calculation in strained GaAs.

Figure 6.4 shows the structure of the first superlattice cathode we studied. The structure parameters of the cathode were similar to the ones reported in Ref. [14]. In this superlattice cathode, strained GaAs layers are sandwiched by GaAsP layers. Both the well and barrier widths are $3 \mathrm{~nm}$. The phosphorus fraction in the GaAsP is 0.36 . A $5 \mathrm{~nm}$ highly-doped GaAs layer is grown on the surface of the superlattice cathode to address the surface-charge-limit problem.

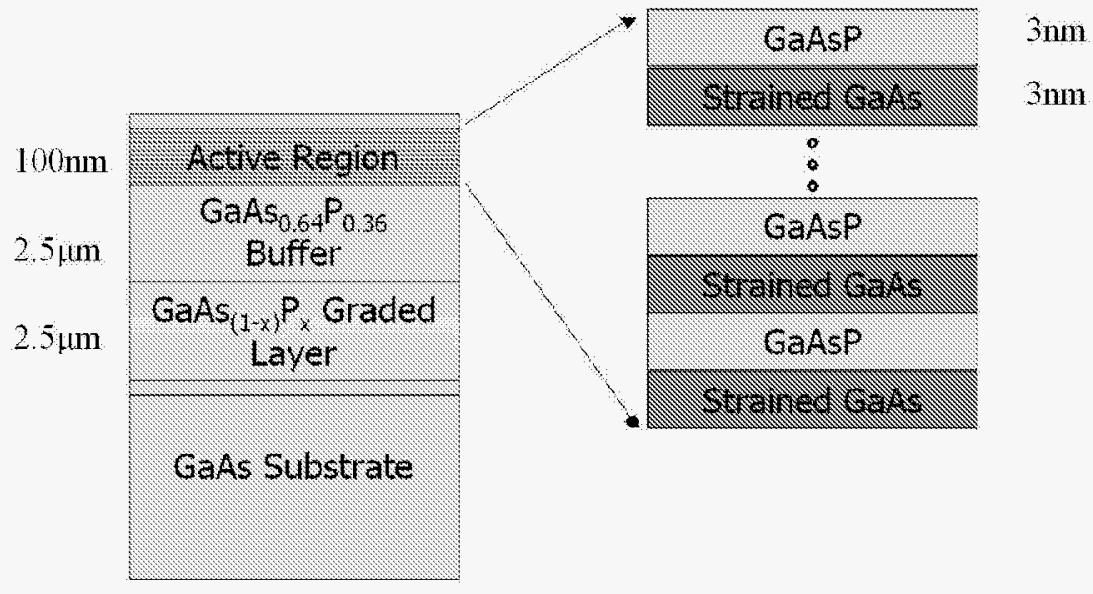

Figure 6.4.

Diagram of epitaxial layers in the grown superlattice photocathode

Figure 4.4 shows the result from a (004) scan in an x-ray diffraction measurement. The experimental results show all the familiar features. The Bragg peaks from GaAs bulk, graded $\mathrm{GaAsP}$, and GaAsP are clearly identified. Additional peaks from the superlattice structure can also be seen. The simulation results show that the barrier width and the well width are about $32 \AA$ with the phosphorus fraction about 0.36 . These numbers are very close to the structure design.

The performance of two superlattice cathodes is shown in Fig. 6.5. Both cathodes exhibited good QE, and their peak polarizations were over $85 \%$. This demonstrated that the high polarization values were reproducible for a given structure across different growth runs. One cathode from the wafer SVT-3984 had been tested in Gun Test Laboratory at SLAC, and there was no surface charge limit observed with available laser energy. 


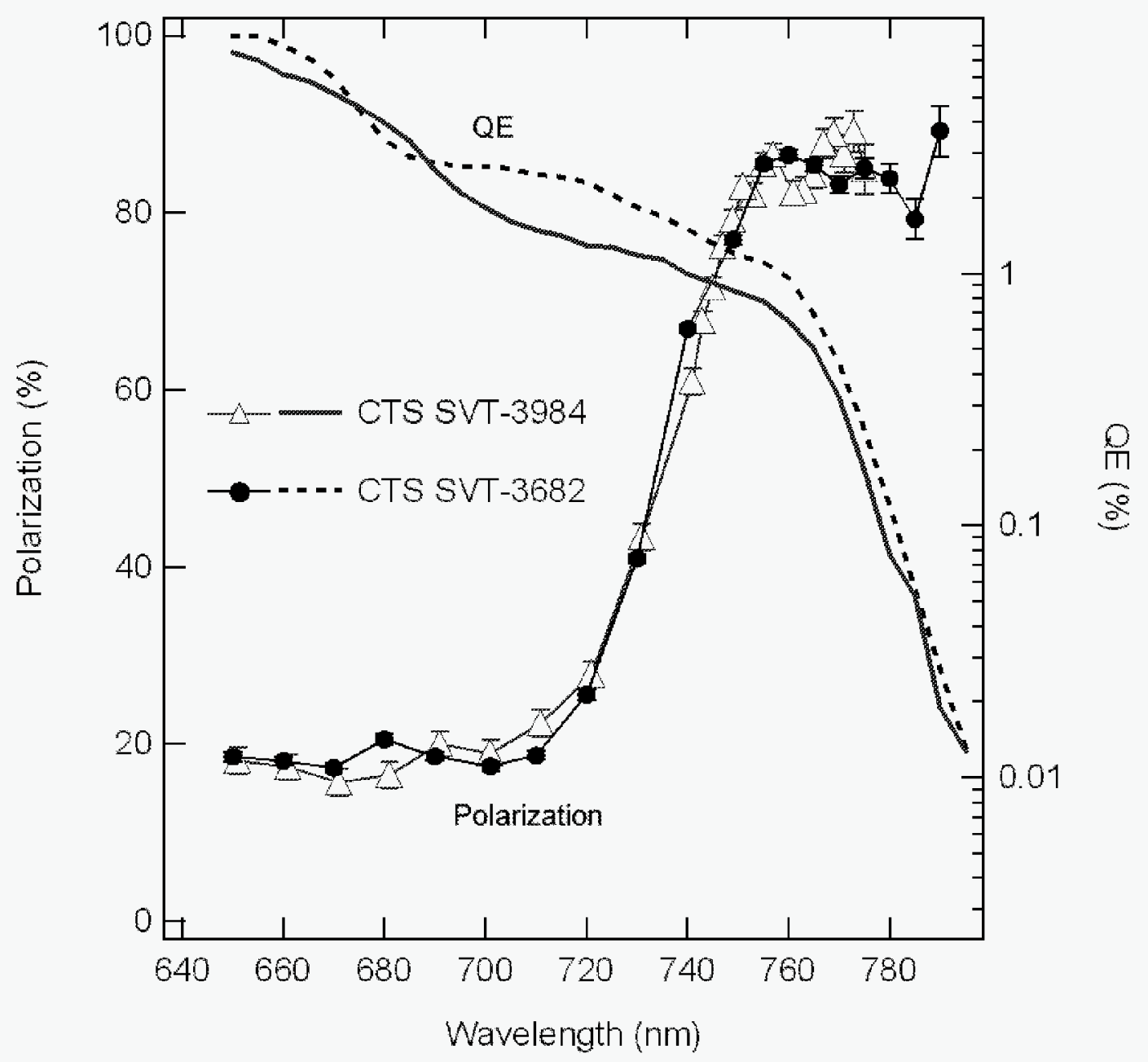

Figure 6.5. Performance of high-gradient-doped superlattice cathodes SVT-3682 and SVT-3984.

Further $x$-ray analysis of these initial samples revealed that the GaAs layers in the $\mathrm{GaAs} / \mathrm{GaAs}_{0.64} \mathrm{P}_{0.36}$ superlattice, which was designed to be pure GaAs, was actually the alloy $\mathrm{GaAs}_{0.96} \mathrm{P}_{0.04}$ (Fig. 6.6). Phosphorus molecules are small and light, and thus are present in the ambient of the growth chamber for up to several minutes after the intentional P-flux was used. With the design of the superlattice, GaAs was always being grown right after a GaAsP layer. Thus, residual phosphorus was present and was being incorporated into the GaAs. In the next growths of the photocathode structure, modifications were made to the growth conditions in an effort to reduce residual $\mathrm{P}$ in the GaAs layers. This included increased the amount of As flux during the GaAs to suppress the $\mathrm{P}$ incorporation, and to add additional pause time between superlattice layers to allow $\mathrm{P}$ to be pumped out. 


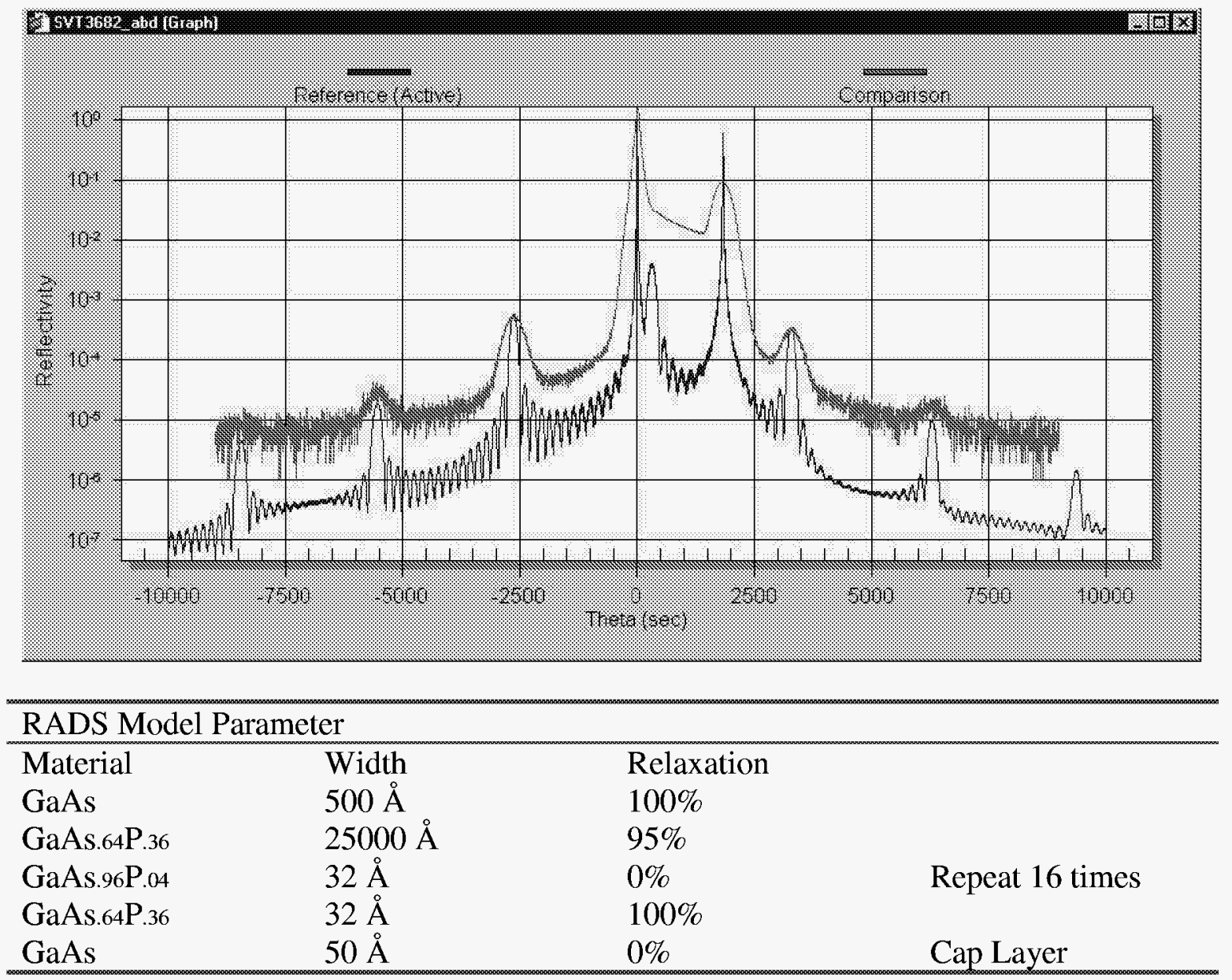

Figure 6.6. X-ray data and simulation confirming that the designed GaAs superlattice layers actually had $4 \% \mathrm{P}$ incorporation.

Photocathode characterization of these superlattice structures revealed that the devices with the thinner $50 \AA$ GaAs cap had higher polarization than samples with a $100 \AA$ GaAs cap. Since highly doped layers are known to cause depolarization, this result was not unexpected. The disadvantage of thinner cap layers is that there is a smaller physical volume available for optical absorption. In order to optimize the polarization value however, $50 \AA \mathrm{GaAs}$ caps will be used for the next structure growths.

A superlattice sample was grown using the same $3 \mathrm{~nm} \mathrm{GaAs} / 3 \mathrm{~nm} \mathrm{GaAs} 0.64 \mathrm{P}_{0.36}$ design (Fig. 6.7), but with modified growth conditions to reduce residual $\mathrm{P}$ incorporation. Photoluminescence of the 2" diameter wafer revealed that the GaAsP buffer contained 35\% phosphorus with less than $1 \%$ variation across the sample (Fig. 6.8). X-ray data and simulation indicated that the residual $\mathrm{P}$ in the GaAs was successfully reduced from $4 \%$ in the initial samples to $1 \%$ in the latest growth (Fig. 6.9). 


\begin{tabular}{|c|c|}
\hline $5 \mathrm{~nm}$ GaAs cap layer & p-type $5 \times 10^{\wedge} 19 / \mathrm{cm} 3$ \\
\hline $3 \mathrm{~nm} \mathrm{GaAs}(0.64) \mathrm{P}(0.36)$ & p-type $5 \times 10 \frown 17 / \mathrm{cm} 3$ \\
\hline $3 \mathrm{~nm}$ GaAs & p-type $5 \times 10^{\wedge} 17 / \mathrm{cm} 3$ \\
\hline $2.5 \mathrm{um} \mathrm{GaAs}(0.64) \mathrm{P}(0.36)$ & p-type $5 \times 10^{\wedge} 18 / \mathrm{cm} 3$ \\
\hline 2.5 um Graded GaAs $(1-\mathrm{x}) \mathrm{P}(\mathrm{x})$ & p-type $5 \times 10 \wedge 18 / \mathrm{cm} 3$ \\
\hline $200 \mathrm{~nm}$ GaAs buffer & p-type $5 \times 10 \wedge 18 / \mathrm{cm} 3$ \\
\hline GaAs substrate & \\
\hline
\end{tabular}

Figure 6.7. Superlattice structure design for photocathode sample \#4182.

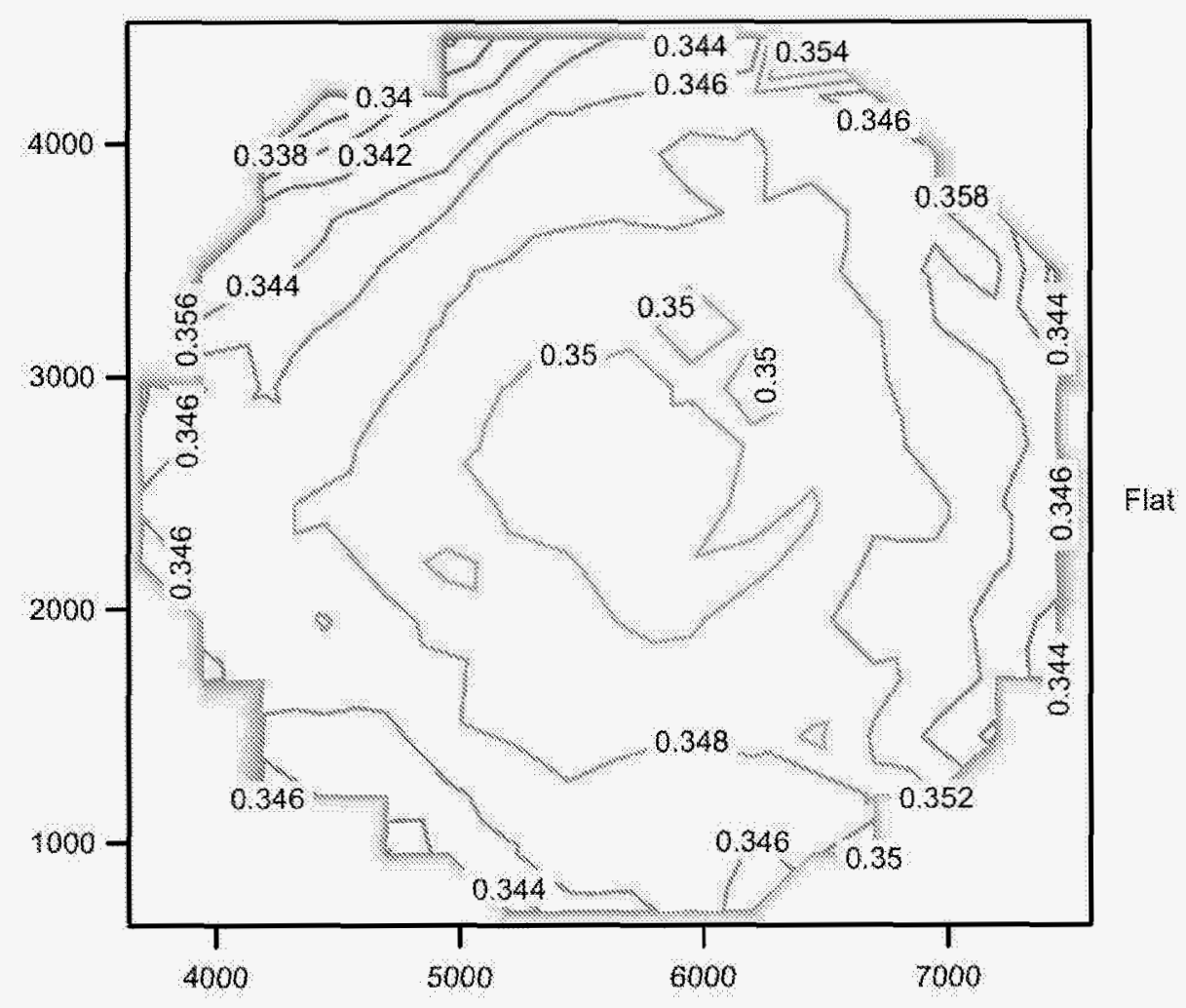

Figure 6.8.

2D map of photoluminescence from the GaAs1-xPx buffer layer of photocathode sample \#4182. . 


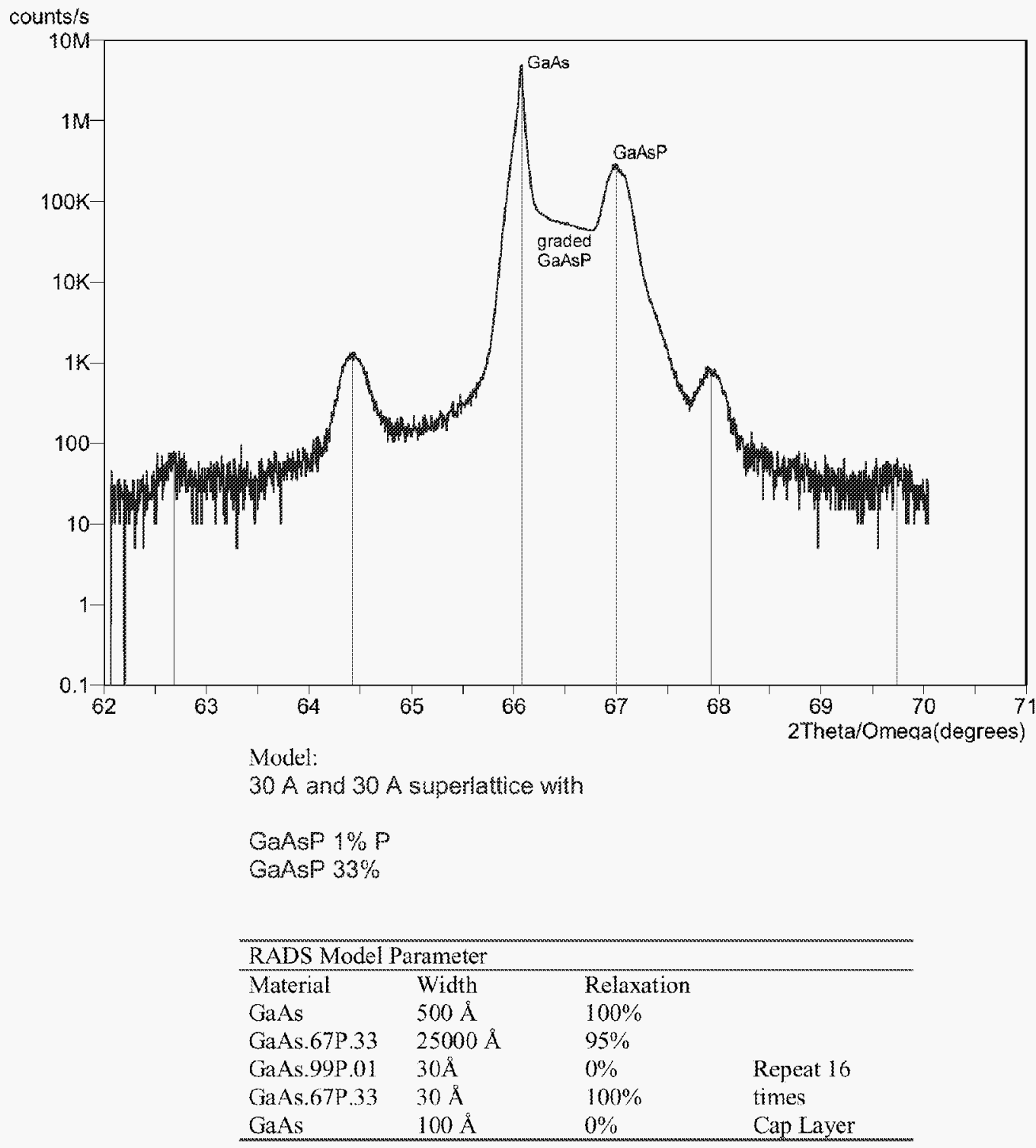

Figure 6.9. X-ray data and model parameters for photocathode sample \#4182. Residual P in the GaAs layers has been reduced to $1 \%$.

Polarization and quantum efficiency measurements of the photocathode \#4182 were performed and plotted alongside the data from the earlier sample \#3682 in Fig. 6.10. The \#4182 sample had a maximum polarization of $86 \%$, comparable to previous samples with the same superlattice design. The quantum efficiency is increased slightly, possibly due to the reduction of residual $P$ in the GaAs layers. 


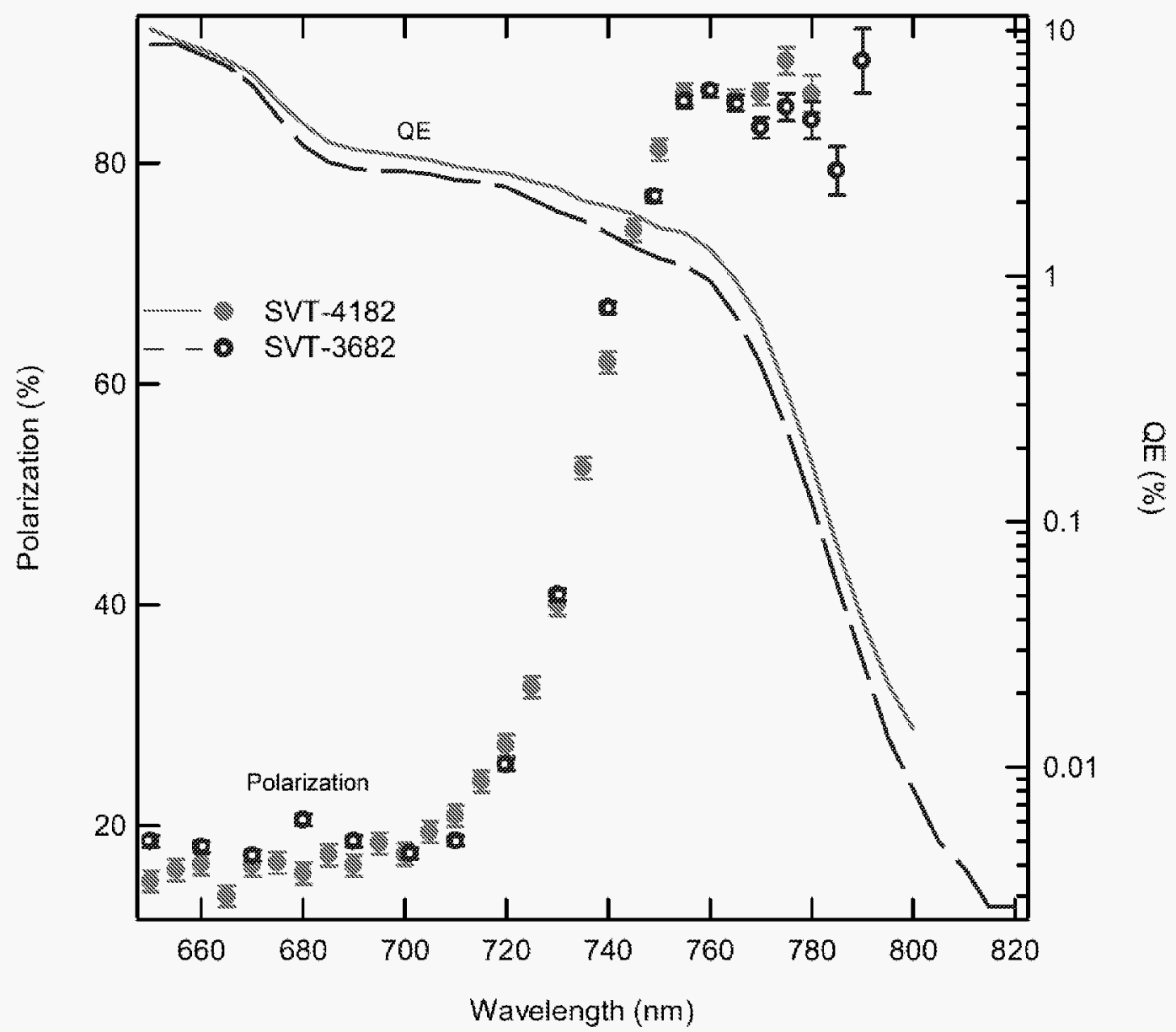

Figure 6.10. Polarization and quantum efficiency of the latest photocathode sample (\#4182) compared with a previous sample of the same structure (\#3682).

\section{PHOTOCATHODE OPTIMIZATION}

High-resolution cross-sectional TEM analysis was also performed on the superlattice. Figure 7.1 shows the low magnification image of the grown structure. Many dislocations were seen in the region where the composition of $\mathrm{GaAs}_{1-\mathrm{x}} \mathrm{P}_{\mathrm{x}}$ is graded from $0<\mathrm{x}<0.36$, which was expected since the lattice constant is changing as the alloy changes. Excellent crystallinity was achieved in the subsequent $\mathrm{GaAs}_{1-\mathrm{x}} \mathrm{P}_{\mathrm{x}}$ buffer layer where the composition remained fixed at $\mathrm{x}=0.36$. 


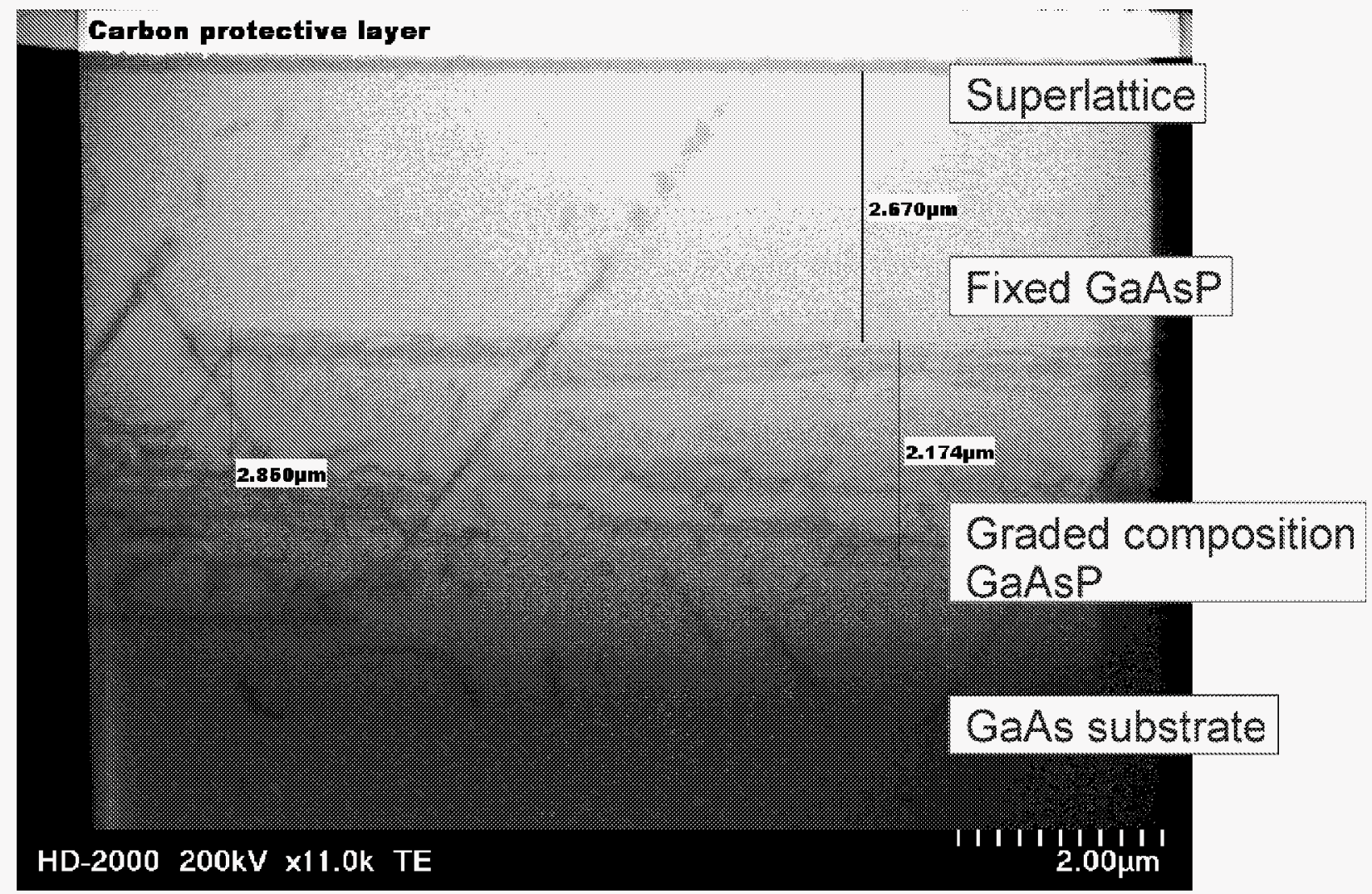

Figure 7.1. Low magnification cross-sectional TEM of the $\mathrm{GaAs}_{1-\mathrm{x}} \mathrm{P}_{\mathrm{x}}$ photocathode superlattice. Dislocations are formed in the compositionally graded region, but become quelled by the fixed composition buffer.

Increasing the magnification, the 16-pair superlattice can be seen (Fig. 7.2). Alternating light and dark areas signify the different materials $\left(\mathrm{GaAs}\right.$ and $\mathrm{GaAs}_{1-\mathrm{x}} \mathrm{P}_{\mathrm{x}}$. Some slight undulations are visible across the surface, possibly due to imperfections in the GaAs substrate surface or artifacts from the TEM sample preparation and measurement technique. Magnifying further, individual atoms can be seen. Interfaces within the superlattice are fairly abrupt, with very small (1-2 monolayers) intermixing of phosphorus atoms bleeding into the GaAs layers. 


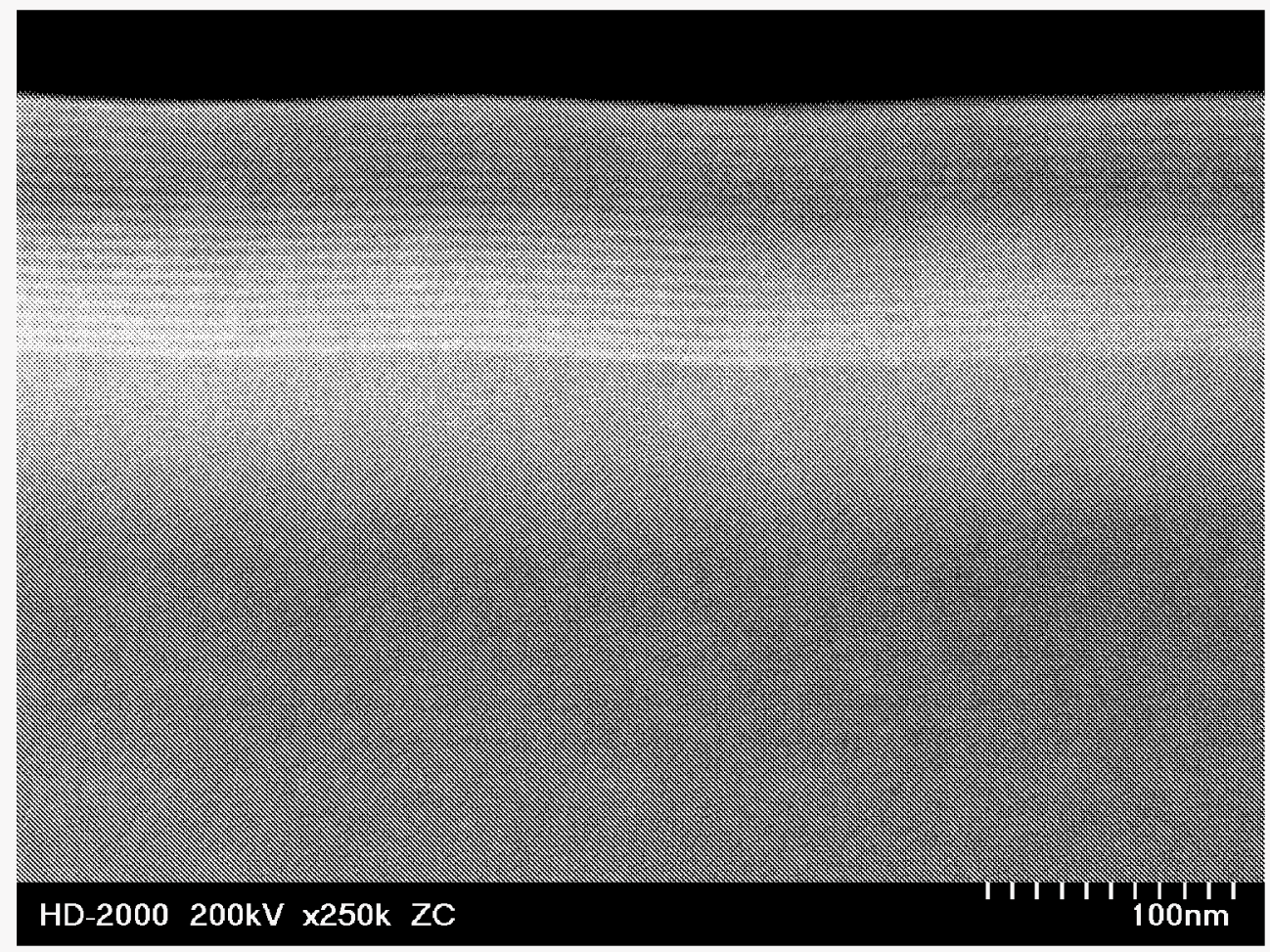

Figure 7.2.

High-resolution crosssectional TEM micrograph of the photocathode structure showing the fixed

$\mathrm{GaAs}_{1-\mathrm{x}} \mathrm{P}_{\mathrm{x}}$ buffer layer and superlattice layer.

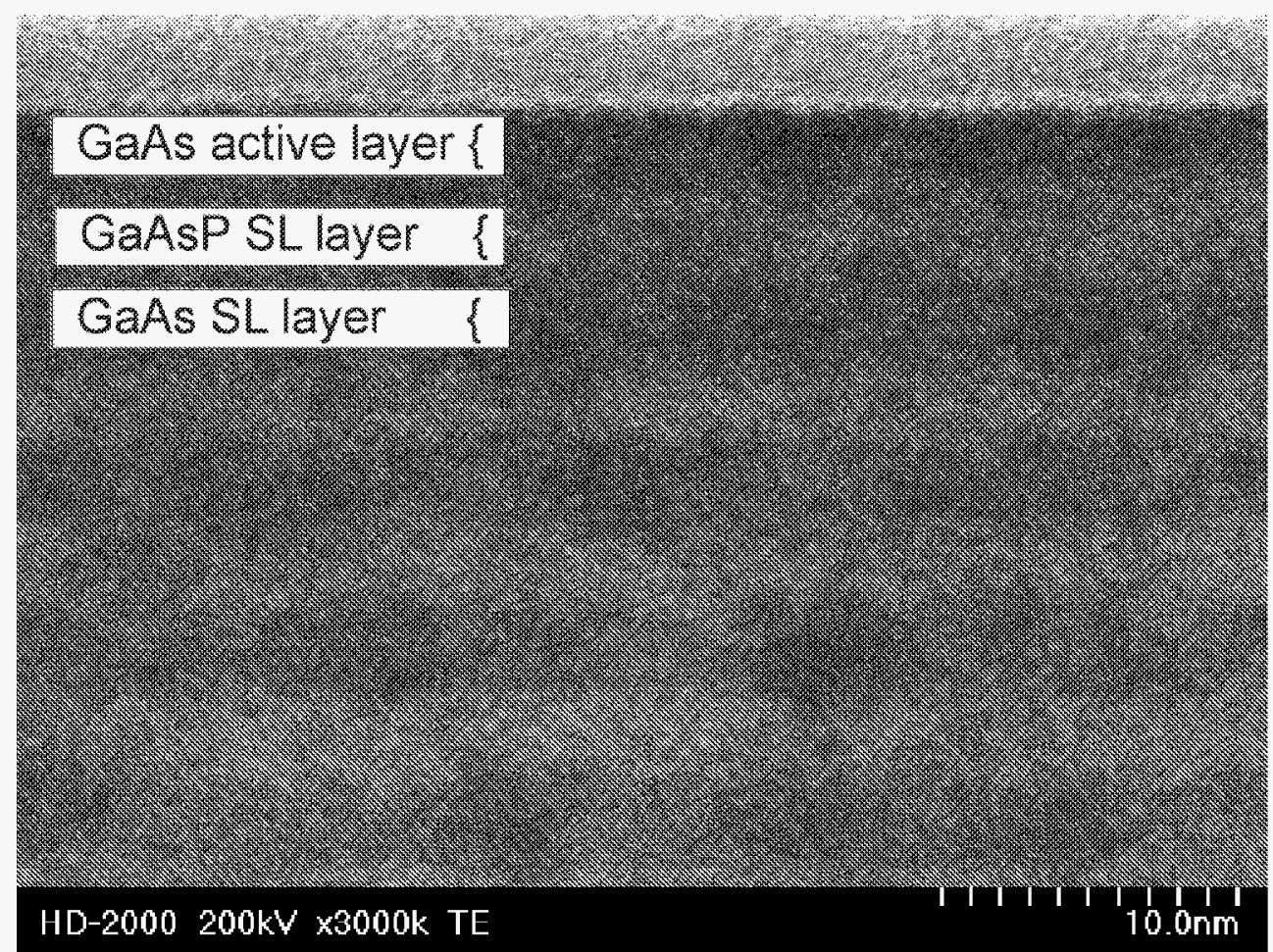

Figure 7.3.

Closeup TEM image of the superlattice and active GaAs cap layer of the photocathode structure 
A series of additional superlattice samples were grown and characterized. Several degrees of freedom were studied to identify trends in device performance versus design. Four parameters specify the superlattice structure - the GaAs well width, the $\mathrm{GaA}_{s 1-x} \mathrm{P}_{x}$ barrier width, the phosphorus fraction, and the number of periods. Table II summarizes eleven superlattice samples studied. In addition to the superlattice samples, a 90-nm thick single strained-layer sample $\left(90 \mathrm{~nm} \mathrm{GaAs} \mathrm{nd}_{0.95} \mathrm{P}_{0.05}\right.$ on $\mathrm{GaAs0.66} \mathrm{P}_{0.34}$ ) was grown for comparison using the same growth method.

TABLE II. Strained GaAs/GaAs1- ${ }_{x} \mathbf{P}_{x}$ superlattice samples.

\begin{tabular}{lcccc}
\hline Sample & Well $(\mathrm{nm})$ & Barrier $(\mathrm{nm})$ & $\mathrm{x}$ & Number of period \\
1 & 4 & 4 & 0.25 & 12 \\
2 & 4 & 4 & 0.30 & 12 \\
3 & 4 & 4 & 0.36 & 12 \\
4 & 4 & 4 & 0.40 & 12 \\
5 & 4 & 4 & 0.36 & 9 \\
6 & 4 & 4 & 0.36 & 15 \\
7 & 4 & 4 & 0.36 & 20 \\
8 & 4 & 4 & 0.36 & 30 \\
9 & 3 & 3 & 0.36 & 16 \\
10 & 4 & 3 & 0.36 & 12 \\
11 & 5 & 3 & 0.36 & \\
\hline
\end{tabular}

The crystallographic structure was analyzed with a double-crystal x-ray diffractometer. Diffraction patterns of (004) symmetric reflection and asymmetric reciprocal-space-maps around (224) reflection were recorded. A dynamic x-ray diffraction software was used to simulate the the diffraction patterns. The phosphorus fraction, the superlattice period and the layer strain were measured. Quantum efficiency (QE) and electron polarization were measured in the SLAC Cathode Test System (CTS) equipped with a load-lock for cathode introduction/removal and a compact medium-energy $(20 \mathrm{kV})$ retarding-field Mott detector for polarization measurements. When excited by linearly polarized light, strained photocathodes show an azimuthal anisotropy in the QE. The QE anisotropy is caused by an asymmetric strain relaxation along the two orthogonal directions [110] and [1 10] [Ref.13]. The measured QE anisotropy is a measure of the overall strain relaxation and can be used to gauge if strain relaxation is causing any depolarization. QE anisotropy is measured at the excitation photon energy that yields a maximum polarization. 
Figure 7.4 shows the polarization and QE as a function of the excitation photon energy for Sample 3 and the reference single strained-layer sample. The peak polarization was $86 \%$ for Sample 3 and $81 \%$ for the strained sample, while the QE at the peak polarization was $1.2 \%$ and $0.3 \%$, respectively. The superlattice-cathode $\mathrm{QE}$ spectrum shows two distinctive steps as expected from the density of states for the two dimensional structure, whereas the cathode-QE spectrum for the single strained-layer cathode, which has a three-dimensional density of states, follows a smooth $\sqrt{E-E g}$ behavior at the band gap energy [13]. The first step corresponds to the heavy-hole $(\mathrm{HH})$ band to the conduction band excitation, while the second step to the lighthole (LH) band to the conduction band. By fitting a step function, the band gap energies of the $\mathrm{HH}$ and LH bands relative to the conduction band were measured to be $E_{H H}=1.58 \mathrm{eV}$ and $E_{L H}=$ $1.66 \mathrm{eV}$, indicated by arrows in Figure 7.4. The energy splitting between the HH- and LH-bands was $82 \mathrm{meV}$. In the single strained-layer sample, the HH-LH splitting was not observed in the QE spectrum. A photoluminescence study performed on single strained-layer samples measured an energy splitting of $55 \mathrm{meV}$ [15], indicating that the energy splitting of the superlattice structure was more than $25 \mathrm{meV}$ larger than that of the single strained-layer structure. The measured QE anisotropy of the superlattice samples was only $1.5 \%$, while that of the single strained-layer sample was $10 \%$.

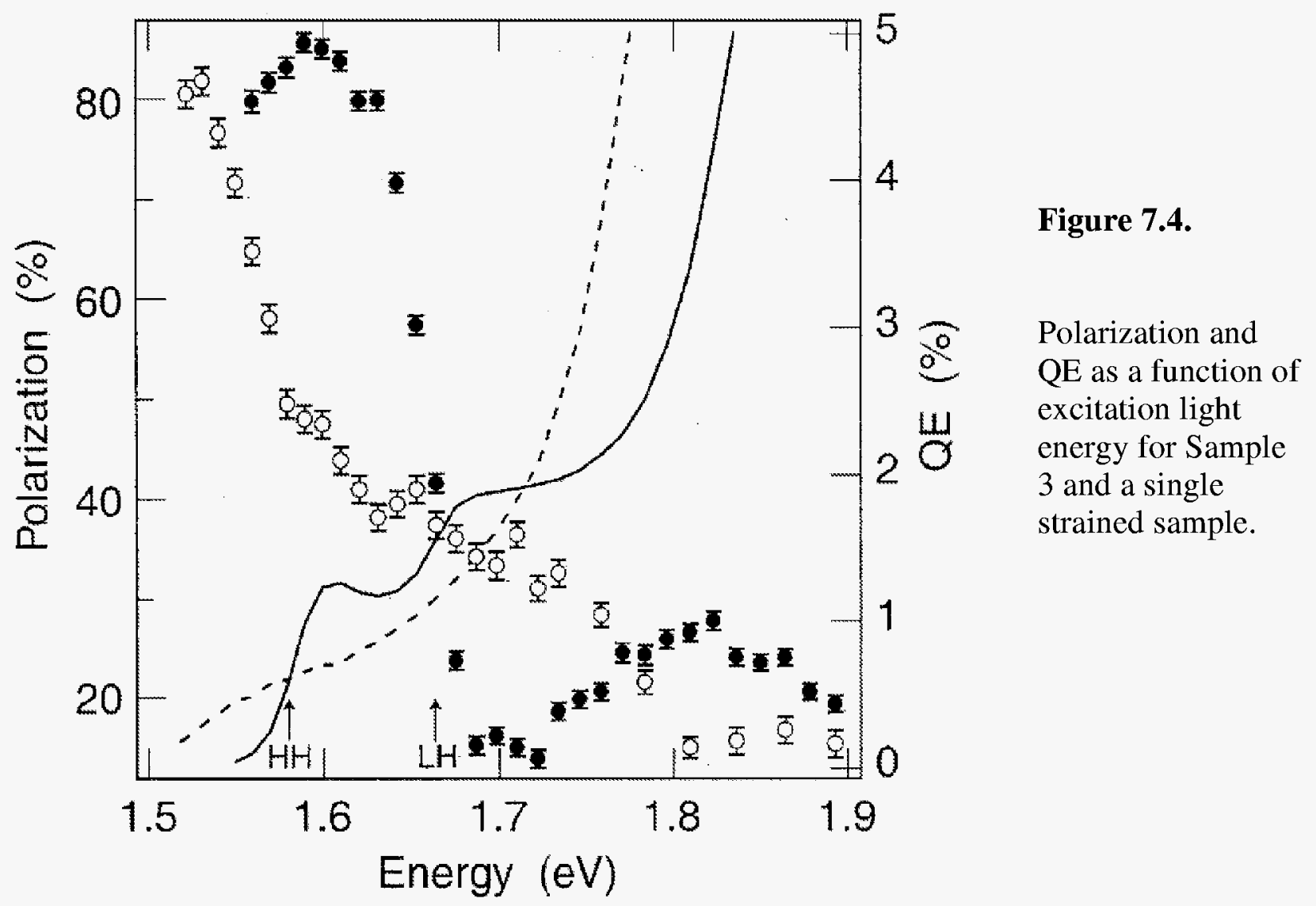


When the phosphorus fraction is varied, the lattice-mismatch between the well and the barrier changes, thus the superlattice strain can be varied. While a larger phosphorus fraction generates a larger strain and therefore a larger energy splitting between the HH and LH bands, the strain within a layer may relax. For Samples 1, 2, 3, and 4, the phosphorus fraction was increased from 0.25 to 0.40 keeping the total superlattice thickness constant. Figure 7.5 shows the peak polarization and QE anisotropy as a function of the phosphorus fraction for constant total thickness. The measured HH-LH energy splitting is also shown in the figure. Although the HH-LH energy splitting increased from $60 \mathrm{meV}$ to $89 \mathrm{meV}$, the peak polarization and the $\mathrm{QE}$ anisotropy did not change significantly at about $85 \%$ and $1.7 \%$, respectively, indicating that this degree of energy splitting is sufficient to maximize the spin polarization.

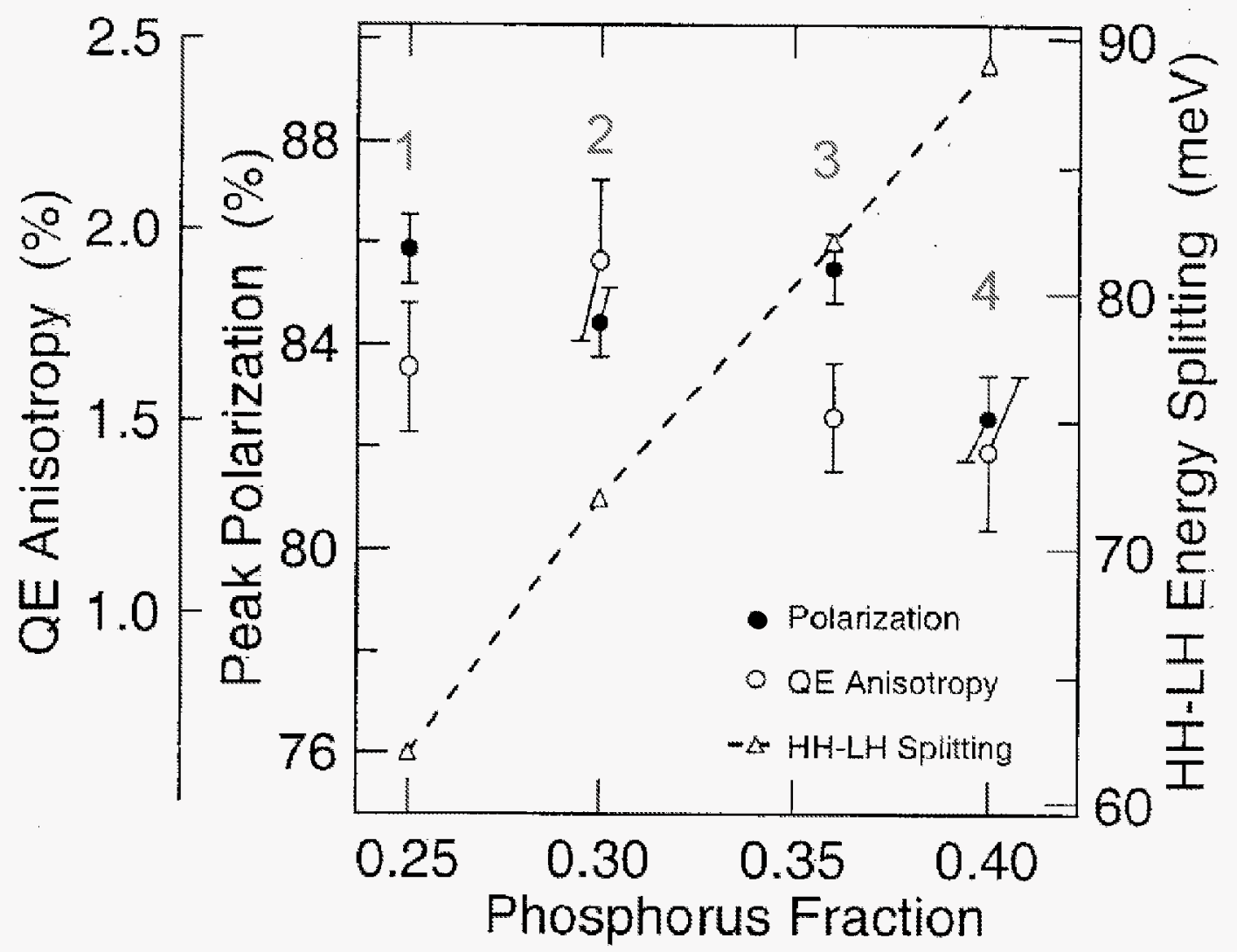

Figure 7.5 Peak polarization (solid circles) and QE anisotropy (open circles) as a function of the phosphorus fraction. The measured HH-LH energy splitting is shown in triangles

Since the well width is smaller than the critical thickness, a significantly thicker superlattice layer was expected to be grown without a strain relaxation. Using samples with the same well $(4 \mathrm{~nm}) /$ barrier $(4 \mathrm{~nm})$ thickness and phosphorus fraction $(\mathrm{x}=0.36)$, the total superlattice thickness was varied. Figure 7.6 shows the peak polarization and QE anisotropy as a function of the number of superlattice period using Samples 3, 5, 6, 7, and 8. Also shown in Figure 7.6 is the strain relaxation in the superlattice GaAs well layers measured using x-ray diffraction. While the strain relaxation steadily increases with the superlattice thickness, the peak polarization and QE anisotropy appear constant at $85.5 \%$, and $1.5 \%$, respectively, for less than 
15 periods. At more than 20 periods however, the peak polarization deceases and the QE anisotropy increases precipitously.

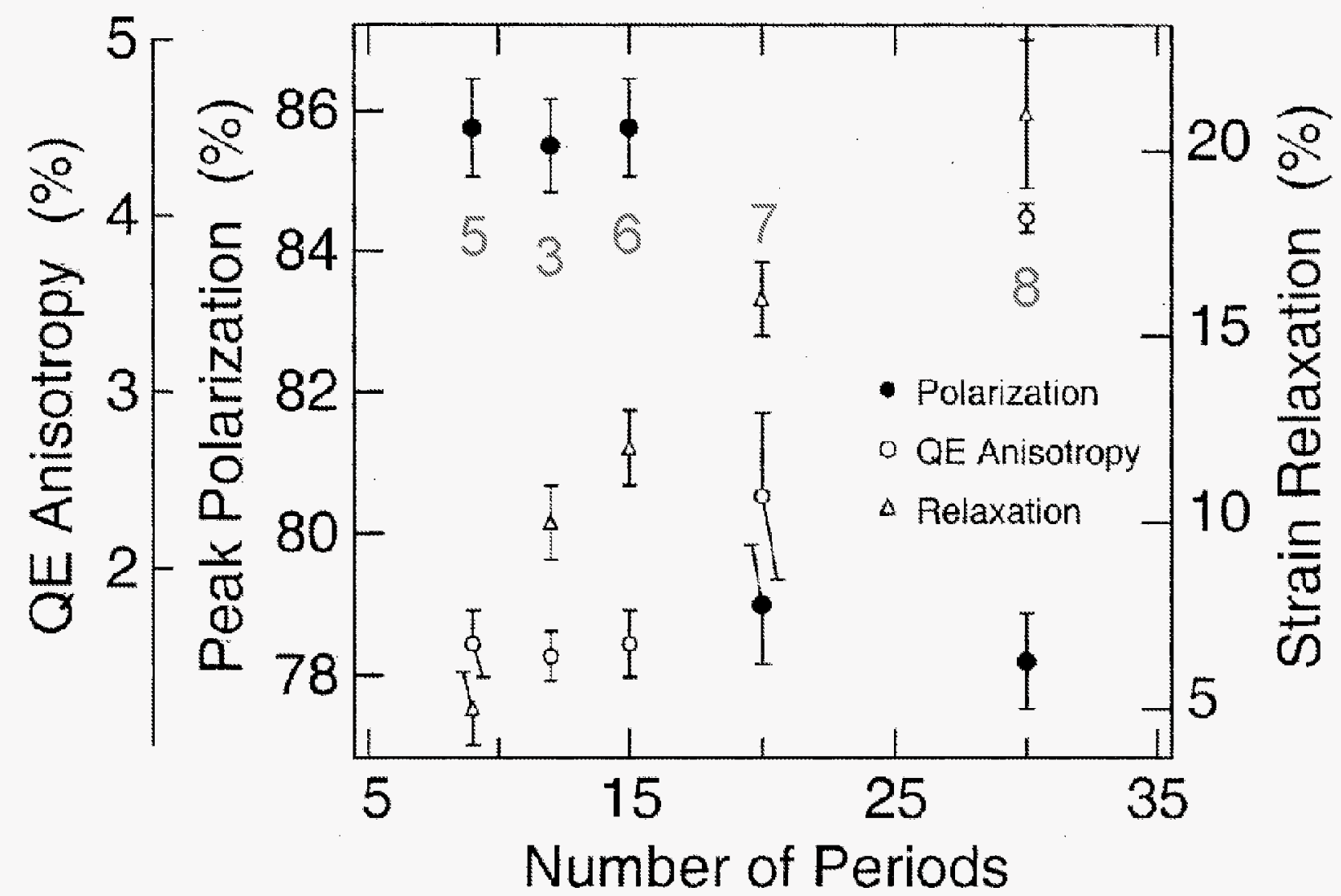

Figure 7.6. Peak polarization and QE anisotropy as a function of the superlattice period

The QE and polarization spectra can be understood in term of the superlattice band structure. In particular, the well width alters the band structure most sensitively. Using samples with the same barrier thickness $(3 \mathrm{~nm})$ and phosphorus fraction $(\mathrm{x}=0.36)$, the well thickness was varied while the number of periods was adjusted to keep the same total superlattice thickness. Figure 7.7 shows the spin polarization as a function of the excitation photon energy for Samples 9, 10, and 11. As the well thickness was increased, the polarization spectrum shifted toward the smaller energy. The peak polarization was, however, independent of the well thickness. The HH energy relative to the conduction band was measured to be $1.61 \mathrm{eV}$ (Sample 9), $1.57 \mathrm{eV}$ (Sample 10), and $1.55 \mathrm{eV}$ (Sample 11). The superlattice band structure was simulated using the transfer matrix method [16]. The calculated HH energies were $1.65,1.61$, and $1.58 \mathrm{eV}$, respectively. The polarization behavior was different at the higher energy. This difference comes from the difference in band structure. 


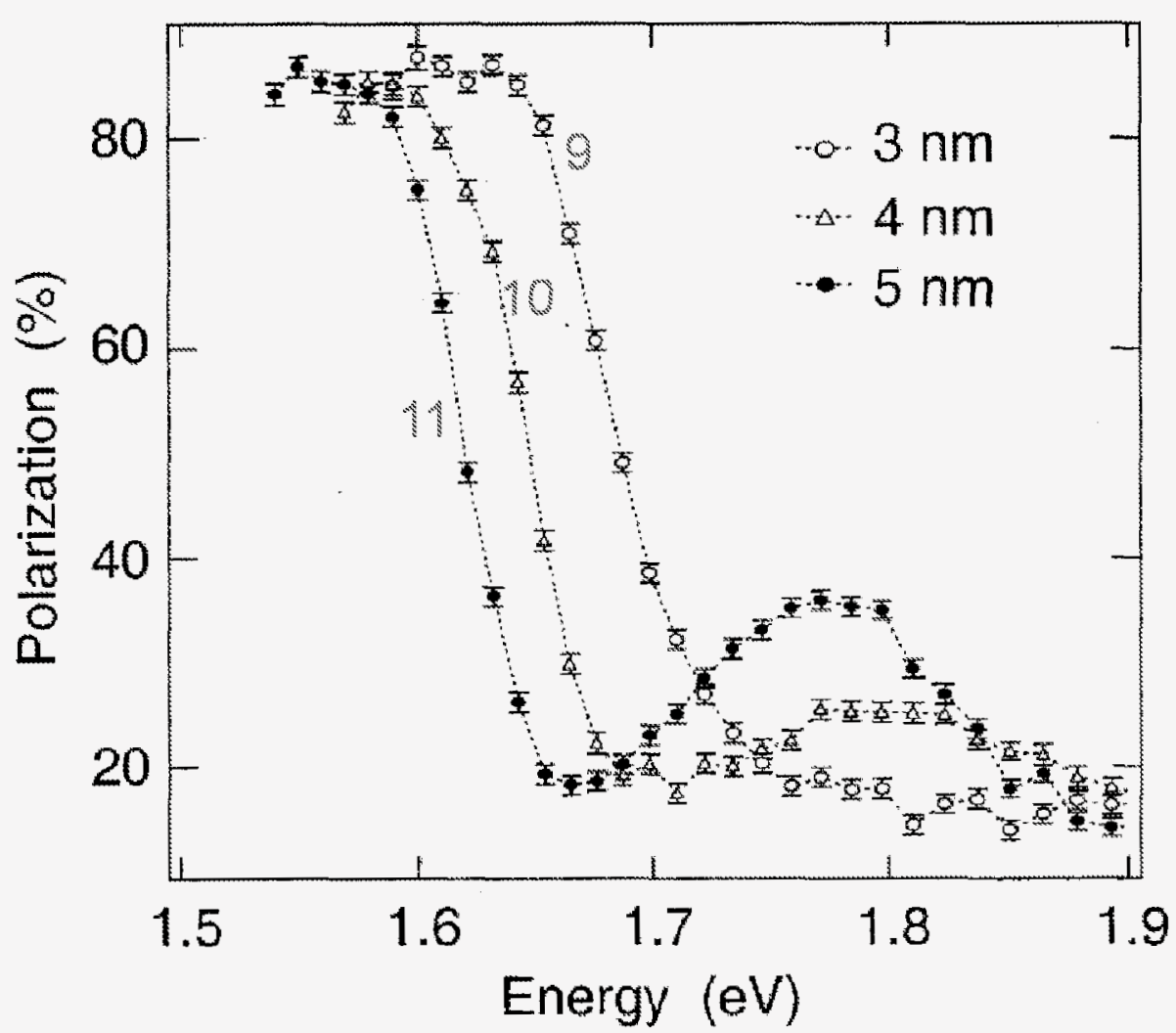

Figure 7.7. Polarization as a function of the excitation light energy for the three superlattice samples with a different well width.

\section{CONCLUSIONS}

SVT Associates and the Stanford Linear Accelerator Center have combined their expertise to promote the operating characteristics for polarized photoemission from cathodes used in advanced high energy physics research. In this program we have realized electron spin polarization as high as $86 \%$ and a record high quantum efficiency in excess of $1 \%$ from strained GaAs/GaAsP superlattice structures. Because the utility of the polarized beam is proportional to the arithmetic square of the efficiency, every percent increase in polarization is significant. The newly developed GaAs/GaAsP strained-superlattice photocathode was installed in the SLAC polarized electron injector in May 2003, and ran successfully for the polarized Moller scattering experiment at 50 $\mathrm{GeV}$ in SLAC End-Station-A. SVTA has also already begun to commercialize the results of this Phase II program by supplying these photocathodes to another domestic research facility.

The polarization appeared saturated at about $86 \%$ and a material-specific spin-depolarization mechanism seemed to be present for the GaAs/GaAsP structure. To address material-specific spin-depolarization and achieve higher polarization, we believe that the strained-superlattice concept can be migrated to materials based on $\mathrm{GaAs} / \mathrm{InGaP}$. We hope to able to demonstrate this further advance in a future effort. 


\section{REFERENCES}

[1] C.Y. Prescott, W.B. Atwood, R.L.A Cottrell, H. DeStaebler, E.L. Garwin, A. Gonidec, R.H. Miller, L.S. Rochester, T. Sato, D.J. Sherden, C.K. Sinclair, S. Stein, R.E. Taylor, J.E. Clendenin, V.W. Hughes, N. Sasao, K.P. Sch“uler, M.G. Borghini, K. L“ubelsmeyer, and W. Jentshke, Phys. Lett. 77B, 347 (1978).

[2] T. Maruyama, E. L. Garwin, R. Prepost, G. H. Zapalac, J. S. Smith, and J. D. Walker, Phys. Rev. Lett. 66, 2376 (1991).

[3] T. Nakanishi, H. Aoyagi, H. Horinaka, Y. Kamiya, T. Kato, S. Nakamura, T. Saka, and M. Tsubata, Phys. Lett. A 158, 345 (1991).

[4] T. Maruyama, E. L. Garwin, R. Prepost, and G. H. Zapalac, Phys. Rev. B46, 4261 (1992).

[5] R. Alley, H. Aoyagi, J. Clendenin, J. Frisch, C. Garden, E. Hoyt, R. Kirby, L. Klaisner, A. Kulikov, R. Miller, G. Mulhollan, C. Prescott, P. Saez, D. Schultz, H. Tang, J. Turner, K. Witte, M. Woods, A.D. Yeremian, M. Zolotorev, Nucl. Instr. and Meth. A 365, 1 (1995).

[6] J. H. van der Merwe and C. A. B. Ball (p. 494) and J. W. Matthews (p. 559), in Eptaxial Growth, edited by J. W. Matthews (Academic Press, New York, 1975).

[7] T. Omori, Y. Kurihara, Y. Takeuchi, M. Yoshioka, T. Nakanishi, S. Okumi, M. Tsubata, M. Tawada, K. Togawa, Y. Tanimoto1, C. Takahashi, T. Baba and M. Mizuta, Jpn. J. Appl. Phys., 33, 5676 (1994).

[8] Yu. Mamaev, A. Subashiev, Yu. Yashin, E. Reichert, P. Dresher, N. Faleev, P. Kop'ev, V. Ustinov, and A. Zhukov, Phys. Low-Dim. Struct. 10/11, 1 (1995).

[9] T. Saka, T. Kato, T. Nakanishi, S. Okumi, K. Togawa, H. Horinaka, T. Matsuyama, and T. Baba, Surface Science 454-456, 1042 (2000).

[10] J.W. Matthews and A.E. Blakeslee, J. Crystal Growth 27, 118 (1974).

[11] G.A. Mulhollan, et al, SLAC-PUB 8585 (2001).

[12] T. Maruyama, A. Brachmann, J. E. Clendenin, T. Desikan, E. L. Garwin, R. E. Kirby, D.-A. Luh, J. Turner, and R. Prepost, Nucl. Instrum. Meth. A492, 199-211(2002).

[13] Shun-Lien Chuang, Physics of Photoelectronic Devices, New York, Wiley, 1995.

[14] T. Nishitani, et al., Proceedings of the $14^{\text {th }}$ International Spin Physics Symposium, Osaka, Japan, 2000, AIP Conference Proceedings 570, 2001, pp. 1021-1023.

[15] R.A. Mair, R. Prepost, H. Tang, E.L. Garwin, T. Maruyama, and G. Mulhollan, Phys. Lett. A212, 231, (1996).

[16] C. Y. P. Chao and S. L. Chuang, Phys. Rev. B48, 11086 (1993). 